\title{
SMOOTH GEOMETRIC EVOLUTIONS OF HYPERSURFACES
}

\author{
CARLO MANTEGAZZA
}

AbStRaCt. We consider the gradient flow associated to the following functionals

$$
\mathcal{F}_{m}(\varphi)=\int_{M} 1+\left|\nabla^{m} \nu\right|^{2} d \mu
$$

The functionals are defined on hypersurfaces immersed in $\mathbb{R}^{n+1}$ via a map $\varphi: M \rightarrow \mathbb{R}^{n+1}$, where $M$ is a smooth closed and connected $n$-dimensional manifold without boundary.

Here $\mu$ and $\nabla$ are respectively the canonical measure and the Levi-Civita connection on the Riemannian manifold $(M, g)$, where the metric $g$ is obtained by pulling back on $M$ the usual metric of $\mathbb{R}^{n+1}$ with the map $\varphi$. The symbol $\nabla^{m}$ denotes the $m$-th iterated covariant derivative and $\nu$ is a unit normal local vector field to the hypersurface.

Our main result is that if the order of derivation $m \in \mathbb{N}$ is strictly larger than the integer part of $n / 2$ then singularities in finite time cannot occur during the evolution.

These geometric functionals are related to similar ones proposed by Ennio De Giorgi, who conjectured for them an analogous regularity result. In the final section we discuss the original conjecture of De Giorgi and some related problems.

\section{INTRODUCTION}

In one of his last papers Ennio De Giorgi conjectured that any compact $n$-dimensional hypersurface in $\mathbb{R}^{n+1}$, evolving by the gradient flow of certain functionals depending on sufficiently high derivatives of the curvature does not develop singularities during the flow ([9, 10], Sec. 5, Conj. 2 - see 10 for an English translation).

This result is central in his program to approximate singular geometric flows with sequences of smooth ones.

Representing hypersurfaces in $\mathbb{R}^{n+1}$ as immersions $\varphi: M \rightarrow \mathbb{R}^{n+1}$, we consider the gradient flow associated to the following functionals

$$
\mathcal{F}_{m}(\varphi)=\int_{M} 1+\left|\nabla^{m} \nu\right|^{2} d \mu
$$

where $\mu$ and $\nabla$ are respectively the canonical measure and the Levi-Civita connection on the Riemannian manifold $(M, g)$, where the metric $g$ is obtained by pulling back on $M$ the usual metric of $\mathbb{R}^{n+1}$ via $\varphi$. We denote with $\nabla^{m}$ the $m$-th iterated covariant derivative and with $\nu$ a unit normal local vector field to the hypersurface. Finally, A and $\mathrm{H}$ are respectively the second fundamental form and the mean curvature of the hypersurface.

These functionals are strictly related to the ones proposed by De Giorgi since, roughly speaking, the derivative of the normal is the curvature of $M$. Though not exactly the same, they can play the same role in the approximation process he suggested. In the end of the paper we discuss some other possible functionals and, in particular, the original De Giorgi conjecture.

Our main result is that if the order of derivation $m \in \mathbb{N}$ is strictly larger than $\left[\frac{n}{2}\right]$ (where $\left[\frac{n}{2}\right]$ denotes the integer part of $n / 2$ ), then singularities cannot occur.

The simplest case $n=1$ and $m=1$ is concerned with curves in the plane evolving by the gradient flow of

$$
\mathcal{F}_{1}(\gamma)=\int_{\mathbb{S} 1} 1+k^{2} d s
$$

Date: November 2, 2018.

1991 Mathematics Subject Classification. Primary 53A07; Secondary 53C21, 35K55.

Key words and phrases. Hypersurface, second fundamental form, gradient flow. 
since the curvature $k$ of a curve $\gamma: \mathbb{S}^{1} \rightarrow \mathbb{R}^{2}$ satisfies $k^{2}=|\nabla \nu|^{2}$. The global regularity in this case was showed by Polden in the papers [30, 31] which have been a starting point for our work. Wen in [38] found results similar to Polden's ones, in considering the flow for $\int_{\mathbb{S}^{1}} k^{2} d s$ of curves with a fixed length.

The very first step in attacking our problem is an analysis of the first variation of the functionals $\mathcal{F}_{m}$, which gives rise to a quasilinear system of partial differential equations on the manifold $M$. The small time existence and uniqueness of a smooth flow is a particular case of a very general result of Polden proven in [24, 31]. Then the long time existence is guaranteed as soon we have suitable a priori estimates on the flow.

In the study of the mean curvature flow of a hypersurface $\varphi: M \times[0, T) \rightarrow \mathbb{R}^{n+1}$,

$$
\frac{\partial \varphi}{\partial t}=-\mathrm{H} \nu=\Delta_{t} \varphi
$$

(which is of second order) via techniques such as varifolds, level sets, viscosity solutions (see [1, 3, 5, 15, 251), the maximum principle is the key tool to get comparison results and estimates on solutions. In our case, even if $m=1$, the first variation and hence the corresponding parabolic problem turns out to be of order higher than two, precisely of order $2 m+2$, so we have to deal with equations of fourth order at least. This fact has the relevant consequence that we cannot employ the maximum principle to get pointwise estimates and to compare two solutions, thus losing a whole bunch of geometric results holding for the mean curvature flow. In particular, we cannot expect that an initially embedded hypersurface remains embedded during the flow, since self-intersections can appear in finite time (an example is given by Giga and Ito in [18]). By these reasons, techniques based on the description of the hypersurfaces as level sets of functions seems of difficult application in this case and therefore we adopt a parametric approach as in the work of Huisken [21].

Despite the large literature on the mean curvature flow, fourth or even higher order flows appeared only recently. Besides the cited works of Polden and Wen, we quote the work of Escher, Mayer and Simonett [14] on the surface diffusion flow (see also the references therein)

$$
\frac{\partial \varphi}{\partial t}=\left(\Delta_{t} \mathrm{H}\right) \nu
$$

and of Simonett [35] on the gradient flow of the Willmore functional (see [39])

$$
\mathcal{W}(\varphi)=\int_{M}|\mathrm{~A}|^{2} d \mu
$$

defined on surfaces immersed in $\mathbb{R}^{3}$. In these papers it is shown the long term existence and convergence of the flow for initial data which are $C^{2, \alpha_{-}}$close to a sphere.

In the article of Chruściel [8], the global existence of a fourth order flow of metrics on a twodimensional Riemannian manifold is applied to construct solutions of Einstein vacuum equations representing an isolated gravitational system, called Robinson-Trautman metrics.

Another problem considered by Polden in [31, 32 is the conformal evolution of a metric $g$ on a two-dimensional manifold $M$ by the gradient flow of the functional

$$
\mathcal{R}(g)=\int_{M} F(R) d \mu
$$

where $R$ is the scalar curvature of $(M, g)$ and $F$ is an even, smooth and strictly convex function. Finally, in a very recent paper [26] Kuwert and Schätzle study the global existence and regularity of the gradient flow of the Willmore functional for general initial data.

Our work borrows from $[8,30,31,32$ the basic idea of using interpolation inequalities as a tool to get a priori estimates.

We want to remark here that a strong motivation for the study of these flows is the fact that, in general, regularity is not shared by second order flows, with the notable exceptions of the evolution by mean curvature of embedded curves in the plane (see [16, 19, 23]) and of convex hypersurfaces (see 21]). So our result opens the possibility to approximate canonically singular flows with smooth ones by singular perturbation arguments (see [9, 10] and Section 9). 
In order to show regularity, a good substitute of the pointwise estimates coming from the maximum principle, are suitable estimates on the second fundamental form in Sobolev spaces, using Gagliardo-Nirenberg interpolation type inequalities for tensors. Since the constants involved in these inequalities depends on the Sobolev constants and these latter on the geometry of the hypersurface where the tensors are defined, before doing estimates we absolutely need some uniform control independent of time on these constants. In [30] these controls are obvious as the constants depend only on the length, on the contrary, much more work is needed in 8 , 26, 32, because of the richer geometry of surfaces.

In our case, we will see that if $m$ is large enough, the functional $\mathcal{F}_{m}$, which decreases during the flow, controls the $L^{p}$ norm of the second fundamental form for some exponent $p$ larger than the dimension. This fact, combined with a universal Sobolev type inequality due to Michael and Simon [28], where the dependence of the constants on the curvature is made explicit, allows us to get an uniform bound on the Sobolev constants of the evolving hypersurfaces and then to obtain time-independent estimates on curvature and all its derivatives in $L^{2}$. These bounds imply in turn the desired pointwise estimates and the long time existence and regularity of the flow.

In the last section we will discuss some possible extensions of our results, some open problems and the related conjectures of De Giorgi.

Acknowledgement. We are grateful to Gerhard Huisken for many discussions about geometric flows during his visit at the Scuola Normale Superiore of Pisa. Moreover, we wish to thank Luigi Ambrosio for his constant encouragement and invaluable help in several occasions.

Our work would have been impossible without the enlightening mathematical insight of Ennio De Giorgi. This paper is dedicated to his memory.

\section{Notation And Preliminaries}

We devote this section to introduce the basic notations and facts about differentiable and Riemannian manifolds we need in the paper, a good reference for this introduction is [17] or the first part of 29].

The main objects of the paper are $n$-dimensional closed hypersurfaces immersed in $\mathbb{R}^{n+1}$, that is, pairs $(M, \varphi)$ where $M$ is an $n$-dimensional smooth manifold, compact, connected with empty boundary, and a smooth map $\varphi: M \rightarrow \mathbb{R}^{n+1}$ such that the rank of $d \varphi$ is everywhere equal to $n$.

The manifold $M$ gets in a natural way a metric tensor $g$ turning it in a Riemannian manifold $(M, g)$, by pulling back the standard scalar product of $\mathbb{R}^{n+1}$ with the immersion map $\varphi$.

Taking local coordinates around $p \in M$ given by a chart $F: \mathbb{R}^{n} \supset U \rightarrow M$, we identify the map $\varphi$ with its expression in coordinates $\varphi \circ F: \mathbb{R}^{n} \supset U \rightarrow \mathbb{R}^{n+1}$, then we have local basis of $T_{p} M$ and $T_{p}^{*} M$, respectively given by vectors $\left\{\frac{\partial}{\partial x_{i}}\right\}$ and covectors $\left\{d x_{j}\right\}$.

We will denote vectors on $M$ by $X=X^{i}$, which means $X=X^{i} \frac{\partial}{\partial x_{i}}$, covectors by $Y=Y_{j}$, that is, $Y=Y_{j} d x_{j}$ and a general mixed tensor with $T=T_{j_{1} \ldots j_{l}}^{i_{1} \ldots i_{k}}$, where the indices refer to the local basis.

Sometimes we will need also to consider tensors along $M$, viewing it as a submanifold of $\mathbb{R}^{n+1}$ via the map $\varphi$, in that case we will use the Greek indices to denote the components of such tensors in the canonical basis $\left\{e_{\alpha}\right\}$ of $\mathbb{R}^{n+1}$, for instance, given a vector field $X$ along $M$, not necessarily tangent, we will have $X=X^{\alpha} e_{\alpha}$.

In all the paper the convention to sum over repeated indices will be adopted.

The inner product on $M$, extended to tensors, is given by

$$
g(T, S)=g_{i_{1} s_{1}} \ldots g_{i_{k} s_{k}} g^{j_{1} z_{1}} \ldots g^{j_{l} z_{l}} T_{j_{1} \ldots j_{l}}^{i_{1} \ldots i_{k}} S_{z_{1} \ldots z_{l}}^{s_{1} \ldots s_{k}}
$$

where $g_{i j}$ is the matrix of the coefficients of the metric tensor in the local coordinates and $g^{i j}$ is its inverse. Clearly, the norm of a tensor is

$$
|T|=\sqrt{g(T, T)} .
$$


The scalar product in $\mathbb{R}^{n+1}$ will be denoted with $\langle\cdot \mid \cdot\rangle$. As the metric $g$ is obtained pulling it back with $\varphi$, we have

$$
g_{i j}(x)=\left\langle\frac{\partial \varphi(x)}{\partial x_{i}} \mid \frac{\partial \varphi(x)}{\partial x_{j}}\right\rangle .
$$

The canonical measure induced by the metric $g$ is given by $\mu=\sqrt{G} \mathcal{L}^{n}$ where $G=\operatorname{det}\left(g_{i j}\right)$ and $\mathcal{L}^{n}$ is the standard Lebesgue measure on $\mathbb{R}^{n}$.

The second fundamental form $\mathrm{A}=h_{i j}$ of $M$ is the 2-tensor defined as follows:

$$
h_{i j}(x)=-\left\langle\nu(x) \mid \frac{\partial^{2} \varphi(x)}{\partial x_{i} \partial x_{j}}\right\rangle,
$$

the mean curvature $\mathrm{H}$ is the trace of $\mathrm{A}$,

$$
\mathrm{H}(x)=g^{i j}(x) h_{i j}(x) .
$$

The induced covariant derivative on $(M, g)$ of a vector field $X$ is given by

$$
\nabla_{j} X^{i}=\frac{\partial}{\partial x_{j}} X^{i}+\Gamma_{j k}^{i} X^{k}
$$

where the Christoffel symbols $\Gamma=\Gamma_{j k}^{i}$ are expressed by the following formula,

$$
\Gamma_{j k}^{i}=\frac{1}{2} g^{i l}\left(\frac{\partial}{\partial x_{j}} g_{k l}+\frac{\partial}{\partial x_{k}} g_{j l}-\frac{\partial}{\partial x_{l}} g_{j k}\right) .
$$

In all the paper the covariant derivative $\nabla T$ of a tensor $T=T_{j_{1} \ldots j_{l}}^{i_{1} \ldots i_{k}}$ will be denoted by $\nabla_{s} T_{j_{1} \ldots j_{l}}^{i_{1} \ldots i_{k}}=$ $(\nabla T)_{s j_{1} \ldots j_{l}}^{i_{1} \ldots i_{k}}$.

With $\nabla^{m} T$ we will mean the $k$-th iterated covariant derivative of a tensor $T$.

We recall that the gradient $\nabla f$ of a function and the divergence $\operatorname{div} X$ of a vector field at a point $p \in(M, g)$ are defined respectively by

$$
g(\nabla f(p), v)=d f_{p}(v) \quad \forall v \in T_{p} M
$$

and

$$
\operatorname{div} X=\text { Trace } \nabla X=\nabla_{i} X^{i}=\frac{\partial}{\partial x_{i}} X^{i}+\Gamma_{i k}^{i} X^{k} .
$$

Notice that considering $M$ as a submanifold of $\mathbb{R}^{n+1}$, if $\left\{e_{i}\right\} \in \mathbb{R}^{n+1}$ is an orthonormal basis of $T_{p} M$ we can express the divergence of $X$ as

$$
\operatorname{div} X(p)=g\left(e_{i}, \nabla_{e_{i}} X\right)=\left\langle e_{i} \mid \nabla_{e_{i}}^{M} X\right\rangle=\nabla_{e_{i}}^{M}\left\langle X \mid e_{i}\right\rangle=\nabla_{e_{i}}\left\langle X \mid e_{i}\right\rangle
$$

where $\nabla^{M}$ denotes the projection on $T_{p} M$ of the covariant derivative of $\mathbb{R}^{n+1}$.

Using this last expression we can define the divergence of a general, not necessarily tangent, vector field $X$ along $M$ as a Riemannian submanifold of $\mathbb{R}^{n+1}$.

Such definition is useful in view of the following tangential divergence formula (see [33], Chap. 2, Sec. 7),

$$
\int_{M} \operatorname{div} X d \mu=\int_{M}\langle\nu \mid X\rangle \mathrm{H} d \mu
$$

holding for every vector field $X$ along $M$.

Notice that the right term is well defined since, by definition (2.1), $\mathrm{H} \nu$ is independent of the choice of the local unit normal $\nu$. Moreover, if $X$ is a tangent vector field we recover the usual divergence theorem

$$
\int_{M} \operatorname{div} X d \mu=0 .
$$

The Laplacian $\Delta T$ of a tensor $T$ is

$$
\Delta T=g^{i j} \nabla_{i} \nabla_{j} T .
$$


The Riemann tensor, the Ricci tensor and the scalar curvature are expressible via the second fundamental form as follows,

$$
\begin{aligned}
\mathrm{R}_{i j k l} & =h_{i k} h_{j l}-h_{i l} h_{j k}, \\
\mathrm{Ric}_{i j} & =\mathrm{H} h_{i j}-h_{i l} g^{l k} h_{k j}, \\
R & =\mathrm{H}^{2}-|\mathrm{A}|^{2} .
\end{aligned}
$$

Hence, the formulas for the interchange of covariant derivatives, which involve the Riemann tensor, become

$$
\begin{gathered}
\nabla_{i} \nabla_{j} X^{s}-\nabla_{j} \nabla_{i} X^{s}=\mathrm{R}_{i j k l} g^{k s} X^{l}=\mathrm{R}_{i j l}^{s} X^{l}=\left(h_{i k} h_{j l}-h_{i l} h_{j k}\right) g^{k s} X^{l}, \\
\nabla_{i} \nabla_{j} Y_{k}-\nabla_{j} \nabla_{i} Y_{k}=\mathrm{R}_{i j k l} g^{l s} Y_{s}=\mathrm{R}_{i j k}^{s} Y_{s}=\left(h_{i k} h_{j l}-h_{i l} h_{j k}\right) g^{l s} Y_{s} .
\end{gathered}
$$

The Codazzi equations

$$
\nabla_{i} h_{j k}=\nabla_{j} h_{i k}=\nabla_{k} h_{i j}
$$

imply the following identity (see [36]) which will be crucial in the sequel,

$$
\Delta h_{i j}=\nabla_{i} \nabla_{j} \mathrm{H}+\mathrm{H} h_{i l} g^{l s} h_{s j}-|\mathrm{A}|^{2} h_{i j} .
$$

Also fundamental will be the Gauss-Weingarten relations

$$
\frac{\partial^{2} \varphi}{\partial x_{i} \partial x_{j}}=\Gamma_{i j}^{k} \frac{\partial \varphi}{\partial x_{k}}-h_{i j} \nu, \quad \frac{\partial}{\partial x_{j}} \nu=h_{j l} g^{l s} \frac{\partial \varphi}{\partial x_{s}},
$$

which easily imply $|\nabla \nu|=|\mathrm{A}|$.

Now we introduce some non standard notation which will be useful for the computations of the following sections.

In all the paper we will write $T * S$, following Hamilton [20], to denote a tensor formed by contraction on some indices of the tensors $T$ and $S$ using the coefficients $g^{i j}$.

Abusing a little the notation, if $T_{1}, \ldots, T_{l}$ is a finite family of tensors (here $l$ is not an index of the tensor $T$ ), with the symbol

we will mean $T_{1} * T_{2} * \cdots * T_{l}$.

$$
\underset{i=1}{\gtrless} T_{i}
$$

We will use the symbol $\mathfrak{p}_{s}\left(T_{1}, \ldots, T_{l}\right)$ for a polynomial in the tensors $T_{1}, \ldots, T_{l}$ and their iterated covariant derivatives with the $*$ product like

$$
\mathfrak{p}_{s}\left(T_{1}, \ldots, T_{l}\right)=\sum_{i_{1}+\cdots+i_{l}=s} c_{i_{1} \ldots i_{l}} \nabla^{i_{1}} T_{1} * \cdots * \nabla^{i_{l}} T_{l},
$$

where the $c_{i_{1} \ldots i_{l}}$ are some real constants.

Notice that every tensor $T_{i}$ must be present in every additive term of $\mathfrak{p}_{s}\left(T_{1}, \ldots, T_{l}\right)$ and there are not repetitions.

We will use instead the symbol $\mathfrak{q}^{s}$ when the tensors involved are all $\mathrm{A}$ or $\nabla \nu$, repetitions are allowed and in every additive term of there must be present every argument of $\mathfrak{q}^{s}$, for instance,

$$
\mathfrak{q}^{s}(\nabla \nu, \mathrm{A})=\sum\left(\underset{k=1}{\circledast} \nabla^{i_{k}}(\nabla \nu) \underset{l=1}{\stackrel{M}{\circledast}} \nabla^{j_{l}} \mathrm{~A}\right) \quad \text { with } N, M \geq 1 .
$$

The order $s$ denotes the sum

$$
s=\sum_{k=1}^{N}\left(i_{k}+1\right)+\sum_{l=1}^{M}\left(j_{l}+1\right) .
$$

Remark 2.1. Supposing that $\mathfrak{q}^{s}$ is completely contracted, that is, there are no free indices and we get a function, then the order $s$ has the following strong geometric meaning: if we consider the family of homothetic immersions $\lambda \varphi: M \rightarrow \mathbb{R}^{n+1}$ for $\lambda>0$, they have associated normal $\nu^{\lambda}$, metric $g^{\lambda}$, connection $\nabla^{\lambda}$ and second form $\mathrm{A}^{\lambda}$ satisfying the following rescaling equations,

$$
\begin{gathered}
\left(\nabla^{\lambda}\right)^{i} \nu^{\lambda}=\nabla^{i} \nu \quad\left(\nabla^{\lambda}\right)^{j} \mathrm{~A}^{\lambda}=\lambda \nabla^{j} \mathrm{~A}, \\
\left(g^{\lambda}\right)_{i j}=\lambda^{2} g_{i j} \quad\left(g^{\lambda}\right)^{i j}=\lambda^{-2} g^{i j} .
\end{gathered}
$$


Then every completely contracted polynomial $\mathfrak{q}^{s}$ in $\nabla \nu$ and A will have the form

$$
\sum\left(\nabla^{i_{1}} \nabla \nu\right) \ldots\left(\nabla^{i_{k}} \nabla \nu\right) \ldots\left(\nabla^{i_{N}} \nabla \nu\right) \nabla^{j_{1}} \mathrm{~A} \ldots \nabla^{j_{l}} \mathrm{~A} \ldots \nabla^{j_{M}} \mathrm{~A} g^{w_{1} z_{1}} \ldots g^{w_{t} z_{t}}
$$

with

$$
s=\sum_{k=1}^{N}\left(i_{k}+1\right)+\sum_{l=1}^{M}\left(j_{l}+1\right)
$$

and since the contraction is total it must be

$$
t=\frac{1}{2}\left(\sum_{k=1}^{N}\left(i_{k}+1\right)+\sum_{l=1}^{M}\left(j_{l}+2\right)\right)
$$

as the sum between the large brackets give the number of covariant indices in the product above. By this argument and the rescaling equations above, we see that $\mathfrak{q}^{s}$ rescales as

$$
\begin{aligned}
\mathfrak{q}^{s}\left(\nabla^{\lambda} \nu^{\lambda}, \ldots, \mathrm{A}^{\lambda}\right) & =\lambda^{M-2 t} \mathfrak{q}^{s}(\nabla \nu, \ldots, \mathrm{A}) \\
& =\lambda^{-\left(\sum_{k=1}^{N}\left(i_{k}+1\right)+\sum_{l=1}^{M}\left(j_{l}+1\right)\right)} \mathfrak{q}^{s}(\nabla \nu, \ldots, \mathrm{A}) \\
& =\lambda^{-s} \mathfrak{q}^{s}(\nabla \nu, \ldots, \mathrm{A}) .
\end{aligned}
$$

By this reason, with a little misuse of language, also when $\mathfrak{q}^{s}$ is not completely contracted, we will say that $s$ is the rescaling order of $\mathfrak{q}^{s}$.

In most of the following computations only the rescaling order and the arguments of the polynomials involved will be important, so we will avoid to make explicit their inner structure.

An example in this spirit, are the following substitutions that we will often apply

$$
\nabla \mathfrak{p}_{s}\left(T_{1}, \ldots, T_{l}\right)=\mathfrak{p}_{s+1}\left(T_{1}, \ldots, T_{l}\right) \quad \text { and } \quad \nabla \mathfrak{q}^{z}(\nabla \nu, \ldots, \mathrm{A})=\mathfrak{q}^{z+1}(\nabla \nu, \ldots, \mathrm{A}) .
$$

We advise the reader that the polynomials $\mathfrak{p}_{s}$ and $\mathfrak{q}^{z}$ could vary from a line to another in a computation by addition of terms with the same rescaling order. Moreover, also the constants could vary between different formulas and from a line to another.

\section{First VARIATION}

Given an immersion $\varphi: M \rightarrow \mathbb{R}^{n+1}$ of a smooth closed hypersurface in $\mathbb{R}^{n+1}$, we consider the following functionals for $m \geq 1$,

$$
\mathcal{F}_{m}(\varphi)=\int_{M} 1+\left|\nabla^{m} \nu\right|^{2} d \mu
$$

where $\nu$ is a local unit normal vector field to $M$ and $\left|\nabla^{m} \nu\right|^{2}$ means $\sum_{\alpha=1}^{n+1}\left|\nabla^{m} \nu^{\alpha}\right|^{2}$. The norm $|\cdot|$, the connection $\nabla$ and the measure $\mu$ are all relative to the Riemannian metric $g$ which is induced on $M$ by $\mathbb{R}^{n+1}$ via the immersion $\varphi$. Notice that these functionals are well defined also without a global unit normal vector field, i. e., $M$ is not orientable, because of the modulus.

In this section we are going to analyze the first variation of these functionals. Actually, computing the exact form can be quite long but for our purposes we need only to study some properties of its structure.

Suppose that we have a one parameter family $\mathcal{I}$ of immersions $\varphi_{t}: M \rightarrow \mathbb{R}^{n+1}$, with $\varphi_{0}=\varphi$, we compute

$$
\delta \mathcal{F}_{m}(\varphi)(\mathcal{I})=\left.\frac{d}{d t} \mathcal{F}_{m}\left(\varphi_{t}\right)\right|_{t=0}=\frac{d}{d t} \int_{M} 1+\left.\left|\nabla^{m} \nu\right|^{2} d \mu_{t}\right|_{t=0}
$$

where clearly the metric $g$, the covariant derivative $\nabla$ and the normal $\nu$ depend on $t$.

Setting $X(p)=\left.\frac{\partial}{\partial t} \varphi_{t}(p)\right|_{t=0}$ we obtain a vector field along $M$ as a submanifold of $\mathbb{R}^{n+1}$ via $\varphi$. It is well known that

$$
\left.\frac{\partial}{\partial t} \mu_{t}\right|_{t=0}=\mathrm{H}\langle\nu \mid X\rangle \mu
$$


so it follows,

$$
\begin{aligned}
\left.\frac{d}{d t} \mathcal{F}_{m}\left(\varphi_{t}\right)\right|_{t=0}= & \int_{M}\left|\nabla^{m} \nu\right|^{2} d\left(\left.\frac{\partial \mu_{t}}{\partial t}\right|_{t=0}\right)+\left.\int_{M} \frac{\partial}{\partial t}\left|\nabla^{m} \nu\right|^{2}\right|_{t=0} d \mu \\
= & \int_{M}\left|\nabla^{m} \nu\right|^{2} \mathrm{H}\langle\nu \mid X\rangle d \mu \\
& +\left.\int_{M} \frac{\partial}{\partial t}\left(g^{i_{1} j_{1}} \ldots g^{i_{m} j_{m}} \nabla_{i_{1} \ldots i_{m}} \nu \nabla_{j_{1} \ldots j_{m}} \nu\right)\right|_{t=0} d \mu
\end{aligned}
$$

Then, we need to compute the derivatives in the last term.

For the metric tensor $g_{i j}$ we have

$$
\begin{aligned}
\frac{\partial}{\partial t} g_{i j} & =\frac{\partial}{\partial t}\left\langle\frac{\partial \varphi}{\partial x_{i}} \mid \frac{\partial \varphi}{\partial x_{j}}\right\rangle \\
& =\left\langle\frac{\partial X}{\partial x_{i}} \mid \frac{\partial \varphi}{\partial x_{j}}\right\rangle+\left\langle\frac{\partial X}{\partial x_{j}} \mid \frac{\partial \varphi}{\partial x_{i}}\right\rangle \\
& =\frac{\partial}{\partial x_{i}}\left\langle X \mid \frac{\partial \varphi}{\partial x_{j}}\right\rangle+\frac{\partial}{\partial x_{j}}\left\langle X \mid \frac{\partial \varphi}{\partial x_{i}}\right\rangle-2\left\langle X \mid \frac{\partial^{2} \varphi}{\partial x_{i} \partial x_{j}}\right\rangle \\
& =a_{i j}(X) .
\end{aligned}
$$

Differentiating the formula $g_{i s} g^{s j}=\delta_{i}^{j}$ we get

$$
\frac{\partial}{\partial t} g^{i j}=-g^{i s} \frac{\partial}{\partial t} g_{s l} g^{l j}=-g^{i s} a_{s l}(X) g^{l j} .
$$

The derivative of the normal $\nu$ is given by

$$
\begin{aligned}
\frac{\partial}{\partial t} \nu & =\left\langle\frac{\partial \nu}{\partial t} \mid \frac{\partial \varphi}{\partial x_{i}}\right\rangle \frac{\partial \varphi}{\partial x_{j}} g^{i j}=-\left\langle\nu \mid \frac{\partial^{2} \varphi}{\partial t \partial x_{i}}\right\rangle \frac{\partial \varphi}{\partial x_{j}} g^{i j} \\
& =-\left\langle\nu \mid \frac{\partial X}{\partial x_{i}}\right\rangle \frac{\partial \varphi}{\partial x_{j}} g^{i j}=-\nabla\langle\nu \mid X\rangle+\left\langle\frac{\partial \nu}{\partial x_{i}} \mid X\right\rangle \frac{\partial \varphi}{\partial x_{j}} g^{i j} \\
& =-\nabla\langle\nu \mid X\rangle+\nabla \nu^{\alpha} X^{\alpha}=b(X) .
\end{aligned}
$$

Finally the derivative of the Christoffel symbols is

$$
\begin{aligned}
\frac{\partial}{\partial t} \Gamma_{j k}^{i}= & \frac{1}{2} g^{i l}\left\{\frac{\partial}{\partial x_{j}}\left(\frac{\partial}{\partial t} g_{k l}\right)+\frac{\partial}{\partial x_{k}}\left(\frac{\partial}{\partial t} g_{j l}\right)-\frac{\partial}{\partial x_{l}}\left(\frac{\partial}{\partial t} g_{j k}\right)\right\} \\
& +\frac{1}{2} \frac{\partial}{\partial t} g^{i l}\left\{\frac{\partial}{\partial x_{j}} g_{k l}+\frac{\partial}{\partial x_{k}} g_{j l}-\frac{\partial}{\partial x_{l}} g_{j k}\right\} \\
= & \frac{1}{2} g^{i l}\left\{\nabla_{j}\left(\frac{\partial}{\partial t} g_{k l}\right)+\nabla_{k}\left(\frac{\partial}{\partial t} g_{j l}\right)-\nabla_{l}\left(\frac{\partial}{\partial t} g_{j k}\right)\right\} \\
& +\frac{1}{2} g^{i l}\left\{\frac{\partial}{\partial t} g_{k z} \Gamma_{j l}^{z}+\frac{\partial}{\partial t} g_{l z} \Gamma_{j k}^{z}+\frac{\partial}{\partial t} g_{j z} \Gamma_{k l}^{z}+\frac{\partial}{\partial t} g_{l z} \Gamma_{j k}^{z}-\frac{\partial}{\partial t} g_{j z} \Gamma_{k l}^{z}-\frac{\partial}{\partial t} g_{k z} \Gamma_{j l}^{z}\right\} \\
& -\frac{1}{2} g^{i s} \frac{\partial}{\partial t} g_{s z} g^{z l}\left\{\frac{\partial}{\partial x_{j}} g_{k l}+\frac{\partial}{\partial x_{k}} g_{j l}-\frac{\partial}{\partial x_{l}} g_{j k}\right\} \\
= & \frac{1}{2} g^{i l}\left\{\nabla_{j}\left(\frac{\partial}{\partial t} g_{k l}\right)+\nabla_{k}\left(\frac{\partial}{\partial t} g_{j l}\right)-\nabla_{l}\left(\frac{\partial}{\partial t} g_{j k}\right)\right\} \\
& +g^{i l} \frac{\partial}{\partial t} g_{l z} \Gamma_{j k}^{z}-g^{i s} \frac{\partial}{\partial t} g_{s z} \Gamma_{j k}^{z} \\
= & \frac{1}{2} g^{i l}\left\{\nabla_{j}\left(\frac{\partial}{\partial t} g_{k l}\right)+\nabla_{k}\left(\frac{\partial}{\partial t} g_{j l}\right)-\nabla_{l}\left(\frac{\partial}{\partial t} g_{j k}\right)\right\} \\
= & \frac{1}{2} g^{i l}\left\{\nabla_{j} a_{k l}(X)+\nabla_{k} a_{j l}(X)-\nabla_{l} a_{j k}(X)\right\} .
\end{aligned}
$$

Notice that all these derivatives are linear in the field $X$, since the $a_{i j}(X)$ and $b(X)$ are such. 
Lemma 3.1. If $a(X)=\frac{\partial}{\partial t} g$ is the tensor defined before, for every covariant tensor $T=T_{i_{1} \ldots i_{l}}$ we have

$$
\frac{\partial}{\partial t} \nabla^{s} T=\nabla^{s} \frac{\partial T}{\partial t}+\mathfrak{p}_{s-1}(T, \nabla a(X))
$$

where the constants in the polynomials $\mathfrak{p}_{s-1}(T, \nabla a(X))$ are universal.

Moreover, if the tensor $T$ is a function $f: M \rightarrow \mathbb{R}^{k}$ the last term $\mathfrak{p}_{s-1}(f, \nabla a(X))$ can be substituted with another polynomial $\widetilde{\mathfrak{p}}_{s-2}(\nabla f, \nabla a(X))$.

Proof. We prove the lemma by induction on $s \geq 1$.

If $s=1$ then

$$
\begin{aligned}
\frac{\partial}{\partial t} \nabla_{j} T_{i_{1} \ldots i_{l}}= & \frac{\partial}{\partial t}\left(\frac{\partial}{\partial x_{j}} T_{i_{1} \ldots i_{l}}-\Gamma_{j i_{z}}^{r} T_{i_{1} \ldots i_{z-1} r i_{z+1} \ldots i_{l}}\right) \\
= & \frac{\partial}{\partial x_{j}} \frac{\partial}{\partial t} T_{i_{1} \ldots i_{l}}-\Gamma_{j i_{z}}^{r} \frac{\partial}{\partial t} T_{i_{1} \ldots i_{z-1} r i_{z+1} \ldots i_{l}} \\
& -\frac{\partial}{\partial t} \Gamma_{j i_{z}}^{r} T_{i_{1} \ldots i_{z-1} r i_{z+1} \ldots i_{l}} \\
= & \nabla \frac{\partial T}{\partial t}+T * \nabla a(X)
\end{aligned}
$$

by the previous computation, hence

$$
\frac{\partial}{\partial t} \nabla T=\nabla \frac{\partial T}{\partial t}+\mathfrak{p}_{0}(T, \nabla a(X))
$$

and the initial case is proved.

Supposing the lemma holds for $s-1$, we have

$$
\begin{aligned}
\frac{\partial}{\partial t} \nabla^{s} T= & \frac{\partial}{\partial t} \nabla\left(\nabla^{s-1} T\right) \\
= & \nabla\left(\frac{\partial}{\partial t} \nabla^{s-1} T\right)+\mathfrak{p}_{0}\left(\nabla^{s-1} T, \nabla a(X)\right) \\
= & \nabla\left(\nabla^{s-1} \frac{\partial T}{\partial t}+\mathfrak{p}_{s-2}(T, \nabla a(X))\right) \\
& +\mathfrak{p}_{0}\left(\nabla^{s-1} T, \nabla a(X)\right) \\
= & \nabla^{s} \frac{\partial T}{\partial t}+\nabla \mathfrak{p}_{s-2}(T, \nabla a(X)) \\
& +\mathfrak{p}_{0}\left(\nabla^{s-1} T, \nabla a(X)\right) \\
= & \nabla^{s} \frac{\partial T}{\partial t}+\mathfrak{p}_{s-1}(T, \nabla a(X))
\end{aligned}
$$

where we set

$$
\mathfrak{p}_{s-1}(T, \nabla a(X))=\nabla \mathfrak{p}_{s-2}(T, \nabla a(X))+\mathfrak{p}_{0}\left(\nabla^{s-1} T, \nabla a(X)\right) .
$$

By this last formula, it is clear that the constants involved are universal. Moreover, if $T$ is a function $f: M \rightarrow \mathbb{R}^{k}$ then the term $\mathfrak{p}_{0}(f, \nabla a(X))$ vanishes and the same formula says that $\mathfrak{p}_{s-1}(f, \nabla a(X))$ does not contain $f$ without being differentiated.

Remark 3.2. In the following we will omit to underline that all the coefficients of the polynomials $\mathfrak{p}_{s}$ and $\mathfrak{q}^{s}$ which will appear are algebraic, that is, they are the result of formal manipulations. In particular, such coefficients are independent of the manifold $(M, g)$ where the tensors are defined. This is crucial in view of the geometry-independent estimates we want to obtain.

Proposition 3.3. The derivative

$$
\left.\frac{\partial}{\partial t}\left(g^{i_{1} j_{1}} \ldots g^{i_{m} j_{m}} \nabla_{i_{1} \ldots i_{m}} \nu \nabla_{j_{1} \ldots j_{m}} \nu\right)\right|_{t=0}
$$


depends only on the vector field $X=\left.\frac{\partial}{\partial t} \varphi_{t}\right|_{t=0}$ and such dependence is linear.

The first variation of $\mathcal{F}_{m}$

$$
\delta \mathcal{F}_{m}(\varphi)(\mathcal{I})=\left.\frac{d}{d t} \mathcal{F}_{m}\left(\varphi_{t}\right)\right|_{t=0}
$$

is a linear function of the field $X$.

Proof. Distributing the derivative in $t$ on the terms of the product, we have seen that the derivatives of the metric coefficients depends linearly on $X$, it lasts to check the derivative of $\nabla_{i_{1} \ldots i_{m}} \nu$. By the last assertion of Lemma 3.1, we have

$$
\frac{\partial}{\partial t} \nabla^{m} \nu=\nabla^{m} \frac{\partial \nu}{\partial t}+\mathfrak{p}_{m-2}(\nabla \nu, \nabla a(X))
$$

and since $\frac{\partial \nu}{\partial t}=b(X)$ we get

$$
\frac{\partial}{\partial t} \nabla^{m} \nu=\nabla^{m} b(X)+\mathfrak{p}_{m-2}(\nabla \nu, \nabla a(X))
$$

which proves the first part of the lemma as $a(X)$ and $b(X)$ are linear in $X$.

The second statement clearly follows by the previous computations and the first part of the lemma.

By this result, we can write $\delta \mathcal{F}_{m}(\varphi)(\mathcal{I})=\delta \mathcal{F}_{m}(\varphi)(X)$. Now we want to prove that actually the first variation depends only on the normal component of the field $X$, that is, $\langle\nu \mid X\rangle$, by linearity, it is clearly sufficient to show that $\delta \mathcal{F}_{m}(\varphi)(X)=0$ for every tangent vector field $X$. By the previous proposition, in order to compute the derivative (3.1) we can choose any family $\mathcal{I}$ of immersions with $X=\left.\frac{\partial}{\partial t} \varphi_{t}\right|_{t=0}$.

Given a vector field $X$ along $M$ as a submanifold of $\mathbb{R}^{n+1}$ which is tangent, there exists a tangent vector field $Y$ on $M$ such that $d \varphi_{p}(Y(p))=X(p)$ for every $p \in M$.

Then we consider the smooth flow $L(p, t): M \times(-\varepsilon, \varepsilon) \rightarrow M$ generated by $Y$ on $M$ as the solution of the ODE's system

$$
\left\{\begin{array}{l}
\frac{\partial}{\partial t} L(p, t)=Y(L(p, t)) \\
L(p, 0)=p
\end{array}\right.
$$

for every $p \in M$ and $t \in(-\varepsilon, \varepsilon)$, and we define $\varphi_{t}(p)=\varphi(L(p, t))$.

Clearly $\varphi_{0}=\varphi$ and

$$
\left.\frac{\partial}{\partial t} \varphi_{t}(p)\right|_{t=0}=\left.d \varphi_{L(p, t)}\left(\frac{\partial}{\partial t} L(p, t)\right)\right|_{t=0}=d \varphi_{p}(Y(p))=X(p),
$$

hence, using the family $\mathcal{I}=\left\{\varphi_{t}\right\}$ we have

$$
\delta \mathcal{F}_{m}(\varphi)(X)=\left.\frac{d}{d t} \mathcal{F}_{m}\left(\varphi_{t}\right)\right|_{t=0}
$$

If $g_{t}$ is the metric tensor on $M$ induced by $\mathbb{R}^{n+1}$ via the immersion $\varphi_{t}$, then the Riemannian manifolds $\left(M, g_{t}\right)$ and $(M, g)$ are isometric for every $t \in(-\varepsilon, \varepsilon)$, being $I(\cdot, t)=\varphi^{-1} \circ \varphi_{t}:\left(M, g_{t}\right) \rightarrow$ $(M, g)$ an isometry between them. Since the functional $\mathcal{F}_{m}$ is invariant by isometry, $\mathcal{F}_{m}\left(\varphi_{t}\right)$ does not depend on $t$ and its derivative is zero.

By the previous discussion we have then the following proposition.

Proposition 3.4. The first variation $\delta \mathcal{F}_{m}(\varphi)(X)$ depends only on $\langle\nu \mid X\rangle$.

This means that we can suppose that $X$ is a normal field in studying $\delta \mathcal{F}_{m}(\varphi)(X)$, hence we can strengthen the previous computations as follows, 


$$
\begin{aligned}
\frac{\partial}{\partial t} g_{i j} & =a_{i j}(X)=-2\left\langle X \mid \frac{\partial^{2} \varphi}{\partial x_{i} \partial x_{j}}\right\rangle=2\langle\nu \mid X\rangle h_{i j} \\
\frac{\partial}{\partial t} g^{i j} & =-g^{i s} \frac{\partial}{\partial t} g_{s l} g^{l j}=-2\langle\nu \mid X\rangle h^{i j} \\
\frac{\partial}{\partial t} \nu & =-\nabla\langle\nu \mid X\rangle \\
\frac{\partial}{\partial t} \Gamma_{j k}^{i} & =g^{i l}\left\{\nabla_{j}\left(\langle\nu \mid X\rangle h_{k l}\right)+\nabla_{k}\left(\langle\nu \mid X\rangle h_{j l}\right)-\nabla_{l}\left(\langle\nu \mid X\rangle h_{j k}\right)\right\} \\
& =\nabla \mathrm{A} *\langle\nu \mid X\rangle+\mathrm{A} * \nabla\langle\nu \mid X\rangle .
\end{aligned}
$$

Supposing $X$ normal, we have immediately the following modification of Lemma 3.1 substituting the tensor $a_{i j}(X)$ with $2\langle\nu \mid X\rangle h_{i j}$.

Lemma 3.5. For every covariant tensor $T=T_{i_{1} \ldots i_{l}}$, we have

$$
\frac{\partial}{\partial t} \nabla^{s} T=\nabla^{s} \frac{\partial T}{\partial t}+\mathfrak{p}_{s}(T, \mathrm{~A},\langle\nu \mid X\rangle)
$$

where in $\mathfrak{p}_{s}(T, \mathrm{~A},\langle\nu \mid X\rangle)$ the derivative $\nabla^{s} T$ does not appear. If $T$ is a function $f: M \rightarrow \mathbb{R}^{k}$

$$
\frac{\partial}{\partial t} \nabla^{s} f=\nabla^{s} \frac{\partial f}{\partial t}+\mathfrak{p}_{s-1}(\nabla f, \mathrm{~A},\langle\nu \mid X\rangle)
$$

and $\mathfrak{p}_{s-1}(\nabla f, \mathrm{~A},\langle\nu \mid X\rangle)$ does not contain $\nabla^{s} f$.

This lemma and the fact that $\frac{\partial \nu}{\partial t}=-\nabla\langle\nu \mid X\rangle$ lead to the following proposition.

Proposition 3.6. Letting $\left\{e_{\alpha}\right\}$ the canonical basis of $\mathbb{R}^{n+1}$ and setting $\nu=\nu^{\alpha} e_{\alpha} \in \mathbb{R}^{n+1}$, we have

$$
\frac{\partial}{\partial t} \nabla_{i_{1} \ldots i_{m}} \nu^{\alpha}=-\nabla_{i_{1} \ldots i_{m}} \nabla^{\alpha}\langle\nu \mid X\rangle+\mathfrak{p}_{m-1}(\nabla \nu, \mathrm{A},\langle\nu \mid X\rangle)
$$

where we denoted with $\nabla^{\alpha}\langle\nu \mid X\rangle$ the $\alpha$ component of the gradient $\nabla\langle\nu \mid X\rangle$ in the canonical basis of $\mathbb{R}^{n+1}$. Moreover, the derivative $\nabla^{m} \nu$ is not present in $\mathfrak{p}_{m-1}(\nabla \nu, \mathrm{A},\langle\nu \mid X\rangle)$.

We are finally ready to compute

$$
\begin{aligned}
\frac{d}{d t} \int_{M} 1+\left.\left|\nabla^{m} \nu\right|^{2} d \mu_{t}\right|_{t=0}= & \int_{M}\left(1+\left|\nabla^{m} \nu\right|^{2}\right) \mathrm{H}\langle\nu \mid X\rangle d \mu \\
& +\int_{M} g^{i_{1} j_{1}} \ldots \frac{\partial}{\partial t} g^{i_{k} j_{k}} \ldots g^{i_{m} j_{m}} \nabla_{i_{1} \ldots i_{m}} \nu \nabla_{j_{1} \ldots j_{m}} \nu d \mu \\
& -2 \int_{M} g^{i_{1} j_{1}} \ldots g^{i_{m} j_{m}} \nabla_{i_{1} \ldots i_{m}} \nabla^{\alpha}\langle\nu \mid X\rangle \nabla_{j_{1} \ldots j_{m}} \nu^{\alpha} d \mu \\
& +2 \int_{M} \nabla^{m} \nu * \mathfrak{p}_{m-1}(\nabla \nu, \mathrm{A},\langle\nu \mid X\rangle) d \mu \\
= & \int_{M}\left(1+\left|\nabla^{m} \nu\right|^{2}\right) \mathrm{H}\langle\nu \mid X\rangle d \mu \\
& +2 m \int_{M} \nabla^{m} \nu * \nabla^{m} \nu * \mathrm{~A}\langle\nu \mid X\rangle d \mu \\
& -2 \int_{M} g^{i_{1} j_{1}} \ldots g^{i_{m} j_{m}} \nabla_{i_{1} \ldots i_{m}} \nabla^{\alpha}\langle\nu \mid X\rangle \nabla_{j_{1} \ldots j_{m}} \nu^{\alpha} d \mu \\
& +\int_{M} \mathfrak{p}_{m-1}\left(\nabla^{m} \nu, \nabla \nu, \mathrm{A},\langle\nu \mid X\rangle\right) d \mu .
\end{aligned}
$$

Now, in order to "carry away" derivatives from $\langle\nu \mid X\rangle$ in the last integral, we integrate by parts with the divergence theorem, "moving" all the derivatives on the other terms of the products. Hence, we can rewrite it as

$$
\int_{M} \mathfrak{p}_{2 m-2}(\nabla \nu, \nabla \nu, \mathrm{A})\langle\nu \mid X\rangle d \mu
$$


which is equal to

$$
\int_{M} \mathfrak{q}^{2 m+1}(\nabla \nu, \mathrm{A})\langle\nu \mid X\rangle d \mu
$$

with the conventions of Section 2 .

Since also the second integral has this form, collecting them together, we obtain

$$
\begin{aligned}
\frac{d}{d t} \int_{M} 1+\left.\left|\nabla^{m} \nu\right|^{2} d \mu_{t}\right|_{t=0}= & \int_{M} \mathrm{H}\langle\nu \mid X\rangle d \mu+\int_{M} \mathfrak{q}^{2 m+1}(\nabla \nu, \mathrm{A})\langle\nu \mid X\rangle d \mu \\
& -2 \int_{M} g^{i_{1} j_{1}} \ldots g^{i_{m} j_{m}} \nabla_{i_{1} \ldots i_{m}} \nabla^{\alpha}\langle\nu \mid X\rangle \nabla_{j_{1} \ldots j_{m}} \nu^{\alpha} d \mu .
\end{aligned}
$$

Finally, we deal with this last term. First, by the divergence theorem it can be transformed in

$$
-2(-1)^{m} \int_{M} \nabla^{\alpha}\langle\nu \mid X\rangle \nabla^{j_{m} \ldots j_{1}} \nabla_{j_{1} \ldots j_{m}} \nu^{\alpha} d \mu,
$$

second, using the tangential divergence formula (2.2), it is equal to

$$
2(-1)^{m} \int_{M}\langle\nu \mid X\rangle \nabla^{\alpha} \nabla^{j_{m} \ldots j_{1}} \nabla_{j_{1} \ldots j_{m}} \nu^{\alpha} d \mu+\int_{M} \mathfrak{q}^{2 m+1}(\nabla \nu, \mathrm{A})\langle\nu \mid X\rangle d \mu,
$$

where the extra term $\mathfrak{q}^{2 m+1}(\nabla \nu, \mathrm{A})\langle\nu \mid X\rangle$, which has a differentiation order lower than the first term, comes from the product with the mean curvature in the tangential divergence formula.

Notice now that the permutation of derivatives introduces additional lower order terms of the form

$$
\int_{M} \mathfrak{q}^{2 m+1}(\nabla \nu, \mathrm{A})\langle\nu \mid X\rangle d \mu
$$

by formulas (2.3), hence we get

$$
2(-1)^{m} \int_{M}\langle\nu \mid X\rangle \nabla^{j_{1}} \nabla_{j_{1}} \ldots \nabla^{j_{m}} \nabla_{j_{m}} \nabla^{\alpha} \nu^{\alpha} d \mu+\int_{M} \mathfrak{q}^{2 m+1}(\nabla \nu, \mathrm{A})\langle\nu \mid X\rangle d \mu
$$

that is,

$$
2(-1)^{m} \int_{M}\langle\nu \mid X\rangle \overbrace{\Delta \Delta \ldots \Delta}^{m \text { times }} \nabla^{\alpha} \nu^{\alpha} d \mu+\int_{M} \mathfrak{q}^{2 m+1}(\nabla \nu, \mathrm{A})\langle\nu \mid X\rangle d \mu .
$$

By Gauss-Weingarten relations (2.5), we have

$$
\nabla^{\alpha} \nu^{\alpha}=\frac{\partial \varphi^{\alpha}}{\partial x_{i}} g^{i j} h_{j l} g^{l s} \frac{\partial \varphi^{\alpha}}{\partial x_{s}}=g^{i j} h_{j l} g^{l s} g_{s i}=g^{i j} h_{j i}=\mathrm{H}
$$

so we conclude

$$
\begin{aligned}
\delta \mathcal{F}_{m}(\varphi)(X)= & \int_{M} \mathrm{H}\langle\nu \mid X\rangle d \mu+\int_{M} \mathfrak{q}^{2 m+1}(\nabla \nu, \mathrm{A})\langle\nu \mid X\rangle d \mu \\
& +2(-1)^{m} \int_{M} \overbrace{\Delta \Delta \ldots \Delta \mathrm{H}}^{m \text { times }} \mathrm{H}\langle\nu \mid X\rangle d \mu \\
= & \int_{M} \mathfrak{q}^{1}(\mathrm{~A})\langle\nu \mid X\rangle d \mu+\int_{M} \mathfrak{q}^{2 m+1}(\nabla \nu, \mathrm{A})\langle\nu \mid X\rangle d \mu \\
& +2(-1)^{m} \int_{M} \overbrace{\Delta \Delta \ldots \Delta}^{m \text { times }} \mathrm{H}\langle\nu \mid X\rangle d \mu .
\end{aligned}
$$

By the previous discussion this formula holds in general for every vector field $X$ along $M$. We summarize all these facts in the following theorem.

Theorem 3.7. For any $m \geq 1$ the first variation of the functional $\mathcal{F}_{m}$ is given by

$$
\delta \mathcal{F}_{m}(\varphi)(X)=\int_{M} \mathrm{E}_{m}(\varphi)\langle\nu \mid X\rangle d \mu
$$


where the function $\mathrm{E}_{m}(\varphi)$ has the form

$$
\mathrm{E}_{m}(\varphi)=2(-1)^{m} \overbrace{\Delta \Delta \ldots \Delta \Delta}^{m \text { times }} \mathrm{H}+\mathfrak{q}^{2 m+1}(\nabla \nu, \mathrm{A})+\mathfrak{q}^{1}(\mathrm{~A}) .
$$

\section{Gradient Flow and Small Time Existence}

Suppose that $\varphi_{0}: M \rightarrow \mathbb{R}^{n+1}$ is smooth immersion of an $n$-dimensional hypersurface $M$ which is compact, connected and has empty boundary.

We look for a smooth function $\varphi: M \times[0, T)$ such that

1. the map $\varphi_{t}=\varphi(\cdot, t): M \rightarrow \mathbb{R}^{n+1}$ is an immersion;

2. the following partial differential equation is satisfied

$$
\frac{\partial \varphi}{\partial t}(p, t)=-\mathrm{E}_{m}\left(\varphi_{t}\right)(p) \nu(p, t) .
$$

If we have a solution, then we say that the hypersurfaces $M_{t}=\left(M, g_{t}\right)$, where $g_{t}$ is the induced metric on $M$, evolve by the gradient flow of the functional $\mathcal{F}_{m}$.

The small time existence of such flow is a slight modification of the following result of Polden (see [31], Thm. 2.5.2, Sec. 2 or [24]).

Theorem 4.1. For any smooth hypersurface immersion $\varphi_{0}: M \rightarrow N$, with $N$ a smooth $(n+1)-$ dimensional Riemannian manifold, there exists a unique solution to the flow problem

$$
\frac{\partial \varphi}{\partial t}=((-1)^{s+1} \overbrace{\Delta \Delta \ldots \Delta}^{s \text { times }} \mathrm{H}+\Phi\left(\varphi, \nu, \mathrm{A}, \nabla \mathrm{A}, \ldots, \nabla^{2 s-1} \mathrm{~A}\right)) \nu
$$

defined on some interval $0 \leq t<T$ and taking $\varphi_{0}$ as its initial value.

Looking at Polden's proof, it is possible to allow the function $\Phi$ to depend also on the metric $g$, moreover the covariant derivatives of the normal $\nu$, using induction and the Gauss-Weingarten relations (2.5), can be expressed in terms of the covariant derivatives of the curvature (see the proof of Lemma 7.5).

Hence, we can conclude that there exists a small time solution of the problem

$$
\frac{\partial \varphi}{\partial t}=((-1)^{m+1} \overbrace{\Delta \Delta \ldots \Delta}^{m \text { times }} H+\Phi\left(\varphi, g, \mathrm{~A}, \nu, \nabla \mathrm{A}, \nabla \nu, \ldots, \nabla^{2 m-1} \mathrm{~A}, \nabla^{2 m} \nu\right)) \nu
$$

which includes our case up to a constant multiplying the leading term. Since such a constant can be eliminated by a time-only rescaling and since a smooth evolution of an immersed compact manifold clearly remains an immersion at least for some positive time, we have a small time existence and uniqueness result for the gradient flow of $\mathcal{F}_{m}$ with every initial hypersurface.

\section{A Priori Estimates}

To prove long time existence we need a priori estimates on the second fundamental form and its derivatives which are obtained via Sobolev and Gagliardo-Nirenberg interpolation inequalities for functions defined on $M_{t}$.

Since the hypersurfaces are moving, also the constants appearing in such inequalities change during the flow, hence, before proceeding with the estimates, we need some uniform control on them.

In this section we see that if the integer $m$ larger than $\left[\frac{n}{2}\right]$ then we have a uniform control, independent of time, on the $L^{n+1}$ norm of the second fundamental form; this is a crucial point where such hypothesis is necessary. This fact will allow us to show in the next section that also the above constants are uniformly bounded during the flow.

In the last part of the section, using an inequality of Michael and Simon, we prove also an a priori lower bound on the volume of the evolving hypersurfaces.

By the very definition of the flow, the value of the functional $\mathcal{F}_{m}$ decreases in time, since

$$
\frac{d}{d t} \mathcal{F}_{m}\left(\varphi_{t}\right)=-\int_{M}\left[\mathrm{E}_{m}\left(\varphi_{t}\right)\right]^{2} d \mu_{t} \leq 0
$$


hence, as long as the flow remains smooth, we have the uniform estimate

$$
\int_{M} 1+\left|\nabla^{m} \mathrm{~A}\right|^{2} d \mu_{t}=\mathcal{F}_{m}\left(\varphi_{t}\right) \leq \mathcal{F}_{m}\left(\varphi_{0}\right)
$$

for every $t \geq 0$.

Now we want to prove that if $m>\left[\frac{n}{2}\right]$, this estimate implies that the $L^{n+1}\left(\mu_{t}\right)$ norms of the second fundamental form $\mathrm{A}$ of $M_{t}$ are uniformly bounded independently of time.

Our starting point is the following universal interpolation type inequalities for tensors.

Proposition 5.1. Suppose that $(M, g)$ is a smooth and compact $n$-dimensional Riemannian manifold without boundary and $\mu$ the measure associated to $g$.

Then for every covariant tensor $T$ and exponents $q \in[1,+\infty)$ and $r \in[1,+\infty]$, we have

$$
\left\|\nabla^{j} T\right\|_{L^{p}(\mu)} \leq C\left\|\nabla^{s} T\right\|_{L^{q}(\mu)}^{\frac{j}{s}}\|T\|_{L^{r}(\mu)}^{\frac{s-j}{s}} \quad \forall j \in[0, s]
$$

with

$$
\frac{1}{p}=\frac{j}{s q}+\frac{s-j}{s r}
$$

where the constant $C$ depends only on $n, s, j, p, q, r$ and not on the metric or the geometry of $M$.

The proof of the case $r=+\infty$ can be found in [20], Sec. 12, along the same lines also the case $r<+\infty$ follows (see also [4, Chap. 3, Sec. 7.6).

Suppose that $M$ is orientable and that $g$ is the metric induced by the immersion $\varphi: M \rightarrow \mathbb{R}^{n+1}$, let $\nu$ be a global unit normal vector field on $M$.

If in (5.2) we consider $T=\nu, s=m, j=1, q=2$ and $r=+\infty$, then we have $|T|=1$ and $p=2 m$, hence

$$
\|\nabla \nu\|_{L^{2 m}(\mu)} \leq C\left\|\nabla^{m} \nu\right\|_{L^{2}(\mu)}^{\frac{1}{m}}
$$

for a constant $C=C(n, m)$.

Since by (2.5) $|\nabla \nu|=|\mathrm{A}|$, we conclude

$$
\int_{M}|\mathrm{~A}|^{2 m} d \mu \leq C \int_{M}\left|\nabla^{m} \nu\right|^{2} d \mu \leq C \mathcal{F}_{m}(\varphi) .
$$

If $M$ is not orientable, then there exists a two-fold Riemannian covering $\widetilde{M}$ of $M$, with a locally isometric projection map $\pi: \widetilde{M} \rightarrow M$ which is orientable and immersed in $\mathbb{R}^{n+1}$ via the map $\varphi \circ \pi$. Repeating the previous argument for $\widetilde{M}$ we get

$$
\int_{\widetilde{M}}|\mathrm{~A}|^{2 m} d \widetilde{\mu} \leq C \int_{\widetilde{M}}\left|\nabla^{m} \nu\right|^{2} d \widetilde{\mu} .
$$

Since $\pi$ is a local isometry and noticing that the global unit normal field on $\widetilde{M}$ gives locally a unit normal field on $M$, all the quantities which appear inside the integrals above do not change passing from $\widetilde{M}$ to $M$, only when we integrate we need to take into account the two-fold structure of the covering. This means that for every smooth function $u: M \rightarrow \mathbb{R}$ we have

$$
\int_{\widetilde{M}} u \circ \pi d \widetilde{\mu}=2 \int_{M} u d \mu \text {. }
$$

Hence, we deduce

$$
2 \int_{M}|\mathrm{~A}|^{2 m} d \mu \leq 2 C \int_{M}\left|\nabla^{m} \nu\right|^{2} d \mu \leq 2 C \mathcal{F}_{m}(\varphi)
$$

which clearly gives the same estimate as in the orientable case.

As $2 m>2\left[\frac{n}{2}\right] \geq n+1$, we have

$$
\int_{M}|\mathrm{~A}|^{n+1} d \mu \leq\left(\int_{M}|\mathrm{~A}|^{2 m} d \mu\right)^{\frac{n+1}{2 m}}(\operatorname{Vol} M)^{\frac{2 m-n-1}{2 m}} \leq C \mathcal{F}_{m}(\varphi)
$$

with a constant $C=C(n, m)$. 
Finally we show that also the volume of $M$ is well controlled by the value of $\mathcal{F}_{m}(\varphi)$ under the hypothesis $m>\left[\frac{n}{2}\right]$.

The bound from above is obvious, the bound from below in dimension $n>1$ can be obtained via the following universal Sobolev inequality due to Michael and Simon (see [28, 33]).

Proposition 5.2. Let $\varphi: M \rightarrow \mathbb{R}^{n+1}$ be an immersion of an n-dimensional, compact hypersurface without boundary. On $M$ we consider the Riemannian metric induced by $\mathbb{R}^{n+1}$ and the corresponding measure $\mu$.

Then, there exists a constant $C=C(n, p)$ depending only on the dimension $n$ and the exponent $p$ such that, for every smooth function $u: M \rightarrow \mathbb{R}$

$$
\left(\int_{M}|u|^{p^{*}} d \mu\right)^{1 / p^{*}} \leq C(n, p)\left(\int_{M}|\nabla u|^{p} d \mu+\int_{M}|\mathrm{H} u|^{p} d \mu\right)^{1 / p},
$$

where $p \in[1, n), n>1$ and $p^{*}=\frac{n p}{n-p}$.

Considering the function $u: M \rightarrow \mathbb{R}$ constantly equal to 1 in the inequality for $p=1$, and taking in account (5.3), we get

$$
\begin{aligned}
(\operatorname{Vol} M)^{\frac{n-1}{n}} & \leq C \int_{M}|\mathrm{H}| d \mu \\
& \leq C\|\mathrm{~A}\|_{L^{n+1}(\mu)}(\operatorname{Vol} M)^{\frac{n}{n+1}} \\
& \leq C \mathcal{F}_{m}(\varphi)^{\frac{1}{n+1}}(\operatorname{Vol} M)^{\frac{n}{n+1}} .
\end{aligned}
$$

Dividing both members by $(\operatorname{Vol} M)^{\frac{n-1}{n}}$, as $\frac{n}{n+1}>\frac{n-1}{n}$ we conclude

$$
1 \leq C \mathcal{F}_{m}(\varphi)^{\frac{1}{n+1}}(\operatorname{Vol} M)^{\frac{1}{n(n+1)}}
$$

that is,

$$
\frac{C}{\mathcal{F}_{m}(\varphi)^{n}} \leq \operatorname{Vol} M \leq \mathcal{F}_{m}(\varphi)
$$

for a constant $C=C(n, m)$.

Remark 5.3. With the same argument, it follows that also $\|\mathrm{A}\|_{L^{n+1}(\mu)}$ can be controlled above and below with $\mathcal{F}_{m}(\varphi)$ and that the functional $\mathcal{F}_{m}$ is uniformly bounded from below by a constant greater than zero.

In the special case $n=1$, we recall that for every closed curve $\gamma: \mathbb{S}^{1} \rightarrow \mathbb{R}^{2}$ in the plane the integral of the modulus of its curvature is at least $2 \pi$, then

$$
2 \pi \leq \int_{\mathbb{S}^{1}}|\mathrm{~A}| d s \leq\left(\int_{\mathbb{S}^{1}}|\mathrm{~A}|^{2} d s\right)^{1 / 2} \sqrt{\text { Length } \gamma} \leq C \sqrt{\mathcal{F}_{m}(\gamma)} \sqrt{\text { Length } \gamma}
$$

Hence,

with $C=C(m)$.

$$
\frac{C}{\mathcal{F}_{m}(\gamma)} \leq \text { Length } \gamma \leq \mathcal{F}_{m}(\gamma)
$$

Putting together all these inequalities and the uniform estimate (5.1) we obtain the following result.

Proposition 5.4. As long as the flow by the gradient of $\mathcal{F}_{m}$ of a hypersurface in $\mathbb{R}^{n+1}$ exists, we have the estimates

$$
\begin{gathered}
\|\mathrm{A}\|_{L^{n+1}\left(\mu_{t}\right)} \leq C_{1}<+\infty \\
0<C_{2} \leq \operatorname{Vol} M_{t} \leq C_{3}<+\infty
\end{gathered}
$$

where the three constants $C_{1}, C_{2}$ and $C_{3}$ are independent of time.

They depend only on $n, m$ and the value of $\mathcal{F}_{m}$ for the initial hypersurface. 


\section{Interpolation Inequalities For Tensors}

As we said, we show now that the uniform bound on the $L^{n+1}$ norm of the second fundamental form implies that the constants involved in some Sobolev and Gagliardo-Nirenberg interpolation type inequalities are also equibounded.

Recalling inequality (5.4), we have

$$
\|u\|_{L^{p^{*}(\mu)}} \leq C(n, p)\left(\|\nabla u\|_{L^{p}(\mu)}+\|\mathrm{H} u\|_{L^{p}(\mu)}\right)
$$

for every $u \in C^{1}(M)$, where $p^{*}=\frac{n p}{n-p}$ and $p \in[1, n)$.

Proposition 6.1. If the manifold $(M, g)$ satisfies $\operatorname{Vol} M+\|\mathrm{H}\|_{L^{n+\delta}(\mu)} \leq B$ for some $\delta>0$ then for every $p \in[1, n)$,

$$
\|u\|_{L^{p^{*}}(\mu)} \leq C\left(\|\nabla u\|_{L^{p}(\mu)}+\|u\|_{L^{p}(\mu)}\right) \quad \forall u \in C^{1}(M),
$$

where the constant $C$ depends only on $n, p, \delta$ and $B$.

Proof. Applying Hölder inequality to the last term of inequality (6.1), we get

$$
\|u\|_{L^{p^{*}}(\mu)} \leq C(n, p)\|\nabla u\|_{L^{p}(\mu)}+C(n, p, \delta, B)\|u\|_{L^{\tilde{p}}(\mu)}
$$

where $\widetilde{p}$ is given by

$$
\widetilde{p}=\frac{p(n+\delta)}{n+\delta-p}=p^{*} \frac{n(n+\delta)}{n(n+\delta)+p^{*} \delta},
$$

then $p<\widetilde{p}<p^{*}$.

Hence, we can interpolate $\|u\|_{L^{\tilde{p}}(\mu)}$ between a small fraction of $\|u\|_{L^{p^{*}(\mu)}}$ and a possibly large multiple of $\|u\|_{L^{p}(\mu)}$,

$$
\|u\|_{L^{p^{*}}(\mu)} \leq C(n, p)\|\nabla u\|_{L^{p}(\mu)}+C(n, p, \delta, B)\left(\varepsilon\|u\|_{L^{p^{*}}(\mu)}+C(\varepsilon, p)\|u\|_{L^{p}(\mu)}\right) .
$$

Choosing $\varepsilon>0$ such that $\varepsilon C(n, p, \delta, B) \leq 1 / 2$ and collecting terms we obtain

$$
\|u\|_{L^{p^{*}}(\mu)} \leq C(n, p, \delta, B)\left(\|\nabla u\|_{L^{p}(\mu)}+\|u\|_{L^{p}(\mu)}\right) .
$$

When $p>n$ we prove the following $L^{\infty}$ result (see also [26], Thm. 5.6).

Proposition 6.2. If the manifold $(M, g)$ satisfies $\mathrm{Vol} M+\|\mathrm{H}\|_{L^{n+\delta}(\mu)} \leq B$ for some $\delta>0$ then for every $p>n$, we have

$$
\max _{M}|u| \leq C\left(\|\nabla u\|_{L^{p}(\mu)}+\|u\|_{L^{p}(\mu)}\right) \quad \forall u \in C^{1}(M),
$$

where the constant $C$ depends only on $n, p, \delta$ and $B$.

Proof. Suppose first that $M$ is embedded and $n+\delta \geq p>n$, clearly $\|\mathrm{H}\|_{L^{p}(\mu)}$ is bounded by a value depending on the constant $B$.

We consider $M$ as a subset of $\mathbb{R}^{n+1}$ via the embedding $\varphi$ and $\mu$ as a measure on $\mathbb{R}^{n+1}$ which is supported on $M$. Then the following result holds ([33], Thm. 17.7): let $B_{\rho}(x)$ be the ball of radius $\rho$ centered at $x$ in $\mathbb{R}^{n+1}$, for every $0<\sigma<\rho<+\infty$ we have

$$
\left(\frac{\mu\left(B_{\sigma}(x)\right)}{\sigma^{n}}\right)^{1 / p} \leq\left(\frac{\mu\left(B_{\rho}(x)\right)}{\rho^{n}}\right)^{1 / p}+C(n, p, \delta, B)\left(\rho^{1-n / p}-\sigma^{1-n / p}\right) .
$$

Hence,

$$
\left(\frac{\mu\left(B_{\sigma}(x)\right)}{\sigma^{n}}\right)^{1 / p} \leq \frac{C_{1}}{\rho^{n / p}}+C_{2} \rho^{1-n / p},
$$

and choosing $\rho=1$, for every $0<\sigma<1$ we get the inequality

$$
\mu\left(B_{\sigma}(x)\right) \leq C(n, p, \delta, B) \sigma^{n} .
$$


Then we need the following formula which is proved in [33], Sec. 18, as a consequence of the tangential divergence formula (2.2).

For every $0<\sigma<\rho<+\infty$ we have

$$
\frac{\int_{B_{\sigma}(x)} u d \mu}{\sigma^{n}} \leq \frac{\int_{B_{\rho}(x)} u d \mu}{\rho^{n}}+\int_{\sigma}^{\rho} \tau^{-n-1} \int_{B_{\tau}(x)} r(|\nabla u|+|u \mathrm{H}|) d \mu(y) d \tau
$$

where $r=|x-y|$ and $u$ is any smooth non negative function.

Noticing that $r \leq \tau$ and using Hölder inequality we estimate

$$
\begin{aligned}
\frac{\int_{B_{\sigma}(x)} u d \mu}{\sigma^{n}} & \leq \frac{\int_{B_{\rho}(x)} u d \mu}{\rho^{n}}+\left(\int_{M}|\nabla u|^{p}+|u \mathrm{H}|^{p} d \mu\right)^{1 / p} \int_{\sigma}^{\rho} \tau^{-n} \mu\left(B_{\tau}(x)\right)^{1-1 / p} d \tau \\
& \leq \int_{B_{1}(x)} u d \mu+C\left(\|\nabla u\|_{L^{p}(\mu)}+\|u \mathrm{H}\|_{L^{p}(\mu)}\right) \int_{\sigma}^{1} \tau^{-n} \tau^{n-n / p} d \tau
\end{aligned}
$$

where in the last passage we set $\rho=1$ used the previous estimate on $\mu\left(B_{\tau}(x)\right)$. The function $\tau^{-n / p}$ is integrable since $p>n$ and we get

$$
\frac{\int_{B_{\sigma}(x)} u d \mu}{\sigma^{n}} \leq \int_{B_{1}(x)} u d \mu+C\left(\|\nabla u\|_{L^{p}(\mu)}+\|u \mathrm{H}\|_{L^{p}(\mu)}\right) \frac{1-\sigma^{1-n / p}}{1-n / p},
$$

now sending $\sigma$ to zero, on the left side we obtain the value of $u(x)$ times $\omega_{n}$ which is the volume of the unit ball of $\mathbb{R}^{n}$, hence

$$
\begin{aligned}
\omega_{n} u(x) & \leq \int_{B_{1}(x)} u d \mu+C\left(\|\nabla u\|_{L^{p}(\mu)}+\|u \mathrm{H}\|_{L^{p}(\mu)}\right) \\
& \leq C(n, p, \delta, B)\left(\|u\|_{L^{1}(\mu)}+\|\nabla u\|_{L^{p}(\mu)}+\|u \mathrm{H}\|_{L^{p}(\mu)}\right) .
\end{aligned}
$$

For a general $u$ we apply this inequality to the function $u^{2}$, thus

$$
\begin{aligned}
u^{2}(x) & \leq C\left(\int_{M}|u|^{2} d \mu+\left(\int_{M}|u \nabla u|^{p} d \mu\right)^{1 / p}+\left(\int_{M}\left|u^{2} \mathrm{H}\right|^{p} d \mu\right)^{1 / p}\right) \\
& \leq C \max _{M}|u|\left(\int_{M}|u| d \mu+\left(\int_{M}|\nabla u|^{p} d \mu\right)^{1 / p}+\left(\int_{M}|u \mathrm{H}|^{p} d \mu\right)^{1 / p}\right) .
\end{aligned}
$$

Since $x \in \mathbb{R}^{n+1}$ was arbitrary we conclude that

$$
\max _{M}|u| \leq C(n, p, \delta, B)\left(\|u\|_{L^{1}(\mu)}+\|\nabla u\|_{L^{p}(\mu)}+\|u \mathrm{H}\|_{L^{p}(\mu)}\right) .
$$

for a constant $C$ depending on $n, p, \delta$ and $B$.

If $M$ is only immersed, we consider the embeddings of $M$ in $\mathbb{R}^{n+1} \times \mathbb{R}^{k}$ given by the map $\varphi \times \varepsilon \psi$ : $M \rightarrow \mathbb{R}^{n+1} \times \mathbb{R}^{k}$, where $\psi: M \rightarrow \mathbb{R}^{k}$ is an embedding of $M$ in some Euclidean space. Then, repeating the previous argument (it is possible since the starting inequalities from 33 hold for embeddings in any $\mathbb{R}^{l}$ ) we will get the same conclusion with a constant $C_{\varepsilon}$. Finally, as $C_{\varepsilon}$ depends only on Vol $M$ and $\mathrm{H}$, and all the geometric quantities converge uniformly when $\varepsilon$ goes to zero, we conclude that the inequality holds also in the immersed case.

Now, given any $p>n$, we choose $\widetilde{p}=\frac{1}{2} \min \{n+p, 2 n+\delta\}$, then clearly $n<\widetilde{p}<\min \{p, n+\delta / 2\}$. By the inequality above we have

$$
\max _{M}|u| \leq C(n, \widetilde{p}, \delta, B)\left(\|u\|_{L^{1}(\mu)}+\|\nabla u\|_{L^{\tilde{p}}(\mu)}+\|u \mathrm{H}\|_{L^{\tilde{p}}(\mu)}\right),
$$

then using Hölder inequality and an interpolation argument as in the proof of Proposition 6.1 we get

$$
\max _{M}|u| \leq C(n, \widetilde{p}, \delta, B)\left(\|u\|_{L^{1}(\mu)}+\|\nabla u\|_{L^{\tilde{p}}(\mu)}+\|u\|_{L^{p}(\mu)}\right) .
$$

Applying again Hölder inequality, as $\widetilde{p}<p$, we conclude that

$$
\max _{M}|u| \leq C(n, \widetilde{p}, \delta, B)\left(\|\nabla u\|_{L^{p}(\mu)}+\|u\|_{L^{p}(\mu)}\right),
$$

which gives the thesis since $\widetilde{p}$ depends only on $n, p$ and $\delta$. 
We now extend these propositions to tensors (see 昍, Prop. 2.11 and also [6, 7]). Since $|T|$ is not necessarily smooth we apply the previous inequalities first to the smooth functions $\sqrt{|T|^{2}+\varepsilon^{2}}$, converging to $|T|$ when $\varepsilon \rightarrow 0$. As

$$
\left|\nabla \sqrt{|T|^{2}+\varepsilon^{2}}\right|=\left|\frac{\langle\nabla T, T\rangle}{\sqrt{|T|^{2}+\varepsilon^{2}}}\right| \leq \frac{|T|}{\sqrt{|T|^{2}+\varepsilon^{2}}}|\nabla T| \leq|\nabla T|
$$

we get then easily the following result.

Proposition 6.3. If the manifold $(M, g)$ satisfies $\mathrm{Vol} M+\|\mathrm{H}\|_{L^{n+\delta}(\mu)} \leq B$ for some $\delta>0$ then for every covariant tensor $T=T_{i_{1} \ldots i_{l}}$ we have,

$$
\begin{array}{rlrl}
\|T\|_{L^{p^{*}(\mu)}} & \leq C\left(\|\nabla T\|_{L^{p}(\mu)}+\|T\|_{L^{p}(\mu)}\right) & & \text { if } 1 \leq p<n, \\
\max _{M}|T| \leq C\left(\|\nabla T\|_{L^{p}(\mu)}+\|T\|_{L^{p}(\mu)}\right) & & \text { if } p>n,
\end{array}
$$

where the constants depend only on $n, l, p, \delta$ and $B$.

We define the Sobolev norm of a tensor $T$ on $(M, g)$ as

$$
\|T\|_{W^{s, q}(\mu)}=\sum_{i=0}^{s}\left\|\nabla^{i} T\right\|_{L^{q}(\mu)} .
$$

Corollary 6.4. In the same hypothesis on $(M, g)$ we have

$$
\begin{array}{cc}
\left\|\nabla^{j} T\right\|_{L^{p}(\mu)} \leq C\|T\|_{W^{s, q}(\mu)} \quad \text { with } \quad \frac{1}{p}=\frac{1}{q}-\frac{s-j}{n}>0, \\
\max _{M}\left|\nabla^{j} T\right| \leq C\|T\|_{W^{s, q}(\mu)} \quad \text { when } \quad & \frac{1}{q}-\frac{s-j}{n}<0 .
\end{array}
$$

The constants depend only on $n, l, s, j, p, q, \delta$ and $B$.

Proof. By inequality (6.2) applied to the tensor $\nabla^{j} T$ we get

$$
\begin{aligned}
\left\|\nabla^{j} T\right\|_{L^{p}(\mu)} & \leq C\left(\left\|\nabla^{j+1} T\right\|_{L^{p_{1}}(\mu)}+\left\|\nabla^{j} T\right\|_{L^{p_{1}}(\mu)}\right) \\
& \leq C\left(\left\|\nabla^{j+2} T\right\|_{L^{p_{2}(\mu)}}+2\left\|\nabla^{j+1} T\right\|_{L^{p_{2}(\mu)}}+\left\|\nabla^{j} T\right\|_{L^{p_{2}(\mu)}}\right) \\
& \leq \quad \cdots \\
& \leq C\left(\left\|\nabla^{s} T\right\|_{L^{p_{s-j}(\mu)}}+\cdots+\left\|\nabla^{j} T\right\|_{L^{p_{s-j}(\mu)}}\right) \\
& \leq C\|T\|_{W^{s, p_{s-j}(\mu)}} .
\end{aligned}
$$

Since the $p_{i}$ are related by

$$
\frac{1}{p_{i}}=\frac{1}{p_{i+1}}-\frac{1}{n}
$$

$p_{0}=p$ and $p_{s-j}=q$, we have

$$
\frac{1}{p}=\frac{1}{p_{s-j}}-\frac{s-j}{n}=\frac{1}{q}-\frac{s-j}{n},
$$

and the first part of the corollary is proved.

The second part follows analogously using also inequality (6.3).

Now we put together this result and the universal inequalities

$$
\left\|\nabla^{j} T\right\|_{L^{p}(\mu)} \leq C\|T\|_{W^{s, q}(\mu)}^{\frac{j}{s}}\|T\|_{L^{r}(\mu)}^{\frac{s-j}{s}},
$$

which are obviously implied by Proposition 5.1, to get the following interpolation type inequalities. 
Proposition 6.5. In the same hypothesis on $(M, g)$ as before, there exist a constant $C$ depending only on $n, l, s, j, p, q, r, \delta$ and $B$, such that for every covariant tensor $T=T_{i_{1} \ldots i_{l}}$, the following inequality hold

$$
\left\|\nabla^{j} T\right\|_{L^{p}(\mu)} \leq C\|T\|_{W^{s, q}(\mu)}^{a}\|T\|_{L^{r}(\mu)}^{1-a},
$$

for all $j \in[0, s], p, q, r \in[1,+\infty)$ and $a \in[j / s, 1]$ with the compatibility condition

$$
\frac{1}{p}=\frac{j}{n}+a\left(\frac{1}{q}-\frac{s}{n}\right)+\frac{1-a}{r} .
$$

If such condition gives a negative value for $p$, the inequality holds for every $p \in[1,+\infty)$ on the left side.

Proof. The cases $a=j / s$ and $a=1$ are inequalities (6.6) and (6.4), respectively, the intermediate cases, when $j / s<a<1$, are obtained immediately by the log-convexity of $\|\cdot\|_{L^{p}(\mu)}$ in $1 / p$, which is a linear function of $a$, and the fact that the right side is exponential in $a$.

If $p$ is negative then $\frac{1}{q}-\frac{s}{n}<0$ and

$$
\frac{1}{q}-\frac{s-j}{n} \leq \frac{j}{n}+a\left(\frac{1}{q}-\frac{s}{n}\right)+\frac{1-a}{r},
$$

hence, the $L^{\infty}$ estimate of inequality (6.5) together with (6.6) gives the inequality for every $p \in[1,+\infty)$.

Remark 6.6. By simplicity, we avoided to discuss in all the section the critical cases of the inequalities, for instance $p=n$ in Proposition 6.3. Actually, for our purposes, we just need to say that in a critical case we can allow any value of $p \in[1,+\infty)$ in the left side of inequalities like (6.7). This can be seen easily, by considering a suitable inequality with a lower integrability exponent on the right side and then applying Hölder inequality.

Putting together the estimates of this section with Proposition 5.4 we obtain the following result.

Proposition 6.7. As long as the flow by the gradient of $\mathcal{F}_{m}$ of a hypersurface in $\mathbb{R}^{n+1}$ exists, for every smooth covariant tensor $T=T_{i_{1} \ldots i_{1}}$ we have the inequalities

$$
\left\|\nabla^{j} T\right\|_{L^{p}(\mu)} \leq C\|T\|_{W^{s, q}(\mu)}^{a}\|T\|_{L^{r}(\mu)}^{1-a},
$$

for all $j \in[0, s], p, q, r \in[1,+\infty)$ and $a \in[j / s, 1]$ with the compatibility condition

$$
\frac{1}{p}=\frac{j}{n}+a\left(\frac{1}{q}-\frac{s}{n}\right)+\frac{1-a}{r} .
$$

If such condition gives a negative value for $p$, the inequality holds for every $p \in[1,+\infty)$ on the left side.

The constant $C$ depends only on $m, n, l, s, j, p, q, r$ and the value of $\mathcal{F}_{m}$ for the initial hypersurface.

\section{Long Time Existence of the Flow}

Suppose that at a certain time $T>0$ the evolving hypersurface develops a singularity, then considering the family $\left\{M_{t}\right\}_{t \in[0, T)}$, we are going to use the time-independent inequalities (6.8) to show that we have uniform estimates

$$
\max _{M_{t}}\left|\nabla^{k} \mathrm{~A}\right| \leq C_{k}<+\infty \quad \forall t \in[0, T)
$$

for all $k \in \mathbb{N}$. We will see that such estimates are in contradiction with the development of a singularity at time $t=T$, hence the flow must be smooth for every positive time.

To this aim we are going to study the evolution of the following integrals,

$$
\int_{M}\left|\nabla^{k} \mathrm{~A}\right|^{2} d \mu_{t}
$$


Remark 7.1. As in the previous sections, we will omit to say in the computations that all the polynomials $\mathfrak{p}_{s}$ and $\mathfrak{q}^{s}$ which will appear are independent of the manifold $(M, g)$ where the tensors are defined.

First we derive the evolution equations for $g, \nu, \Gamma_{j k}^{i}$ and A. Essentially repeating the computations of Section 3, we get

$$
\begin{aligned}
\frac{\partial}{\partial t} g_{i j} & =-2 \mathrm{E}_{m} h_{i j} \\
\frac{\partial}{\partial t} g^{i j} & =2 \mathrm{E}_{m} h^{i j} \\
\frac{\partial}{\partial t} \nu & =\nabla \mathrm{E}_{m} \\
\frac{\partial}{\partial t} \Gamma_{j k}^{i} & =\nabla \mathrm{E}_{m} * \mathrm{~A}+\mathrm{E}_{m} * \nabla \mathrm{A} .
\end{aligned}
$$

Lemma 7.2. The second fundamental form of $M_{t}$ satisfies the evolution equation

$$
\frac{\partial}{\partial t} h_{i j}=2(-1)^{m} \overbrace{\Delta \circ \ldots \circ \Delta}^{m+1} h_{i j}+\mathfrak{q}^{2 m+3}(\mathrm{~A}, \mathrm{~A})+\mathfrak{q}^{2 m+3}(\nabla \nu, \mathrm{A})+\mathfrak{q}^{3}(\mathrm{~A}) .
$$

Proof. Keeping in mind the Gauss-Weingarten relations (2.5) and the equations above, we compute

$$
\begin{aligned}
\frac{\partial}{\partial t} h_{i j}= & -\frac{\partial}{\partial t}\left\langle\nu \mid \frac{\partial^{2} \varphi}{\partial x_{i} \partial x_{j}}\right\rangle \\
= & \left\langle\nu \mid \frac{\partial^{2}\left(\mathrm{E}_{m} \nu\right)}{\partial x_{i} \partial x_{j}}\right\rangle-\left\langle\nabla \mathrm{E}_{m} \mid \frac{\partial^{2} \varphi}{\partial x_{i} \partial x_{j}}\right\rangle \\
= & \frac{\partial^{2} \mathrm{E}_{m}}{\partial x_{i} \partial x_{j}}+\mathrm{E}_{m}\left\langle\nu \mid \frac{\partial}{\partial x_{i}}\left(h_{j l} g^{l s} \frac{\partial \varphi}{\partial x_{s}}\right)\right\rangle \\
& -\left\langle\frac{\partial \mathrm{E}_{m}}{\partial x_{l}} \cdot \frac{\partial \varphi}{\partial x_{s}} g^{l s} \mid \Gamma_{i j}^{k} \frac{\partial \varphi}{\partial x_{k}}-h_{i j} \nu\right\rangle \\
= & \frac{\partial^{2} \mathrm{E}_{m}}{\partial x_{i} \partial x_{j}}-\Gamma_{i j}^{k} \frac{\partial \mathrm{E}_{m}}{\partial x_{k}}+\mathrm{E}_{m} h_{j l} g^{l s}\left\langle\nu \mid \Gamma_{i s}^{z} \frac{\partial \varphi}{\partial x_{z}}-h_{i s} \nu\right\rangle \\
= & \nabla_{i} \nabla_{j} \mathrm{E}_{m}-\mathrm{E}_{m} h_{i s} g^{s l} h_{l j} .
\end{aligned}
$$

Expanding $\mathrm{E}_{m}$ we continue,

$$
\begin{aligned}
\frac{\partial}{\partial t} h_{i j}= & \nabla_{i} \nabla_{j}(2(-1)^{m} \overbrace{\Delta \Delta \ldots \Delta}^{m \text { times }} \mathrm{H}+\mathfrak{q}^{2 m+1}(\nabla \nu, \mathrm{A})+\mathfrak{q}^{1}(\mathrm{~A})) \\
& -(2(-1)^{m} \overbrace{\Delta \Delta \ldots \Delta}^{m \text { times }} \mathrm{H}+\mathfrak{q}^{2 m+1}(\nabla \nu, \mathrm{A})+\mathfrak{q}^{1}(\mathrm{~A})) h_{i s} g^{s l} h_{l j} \\
= & 2(-1)^{m} \nabla_{i} \nabla_{j} \overbrace{\Delta \Delta \ldots \Delta}^{m \text { times }} \mathrm{H}+\mathfrak{q}^{2 m+3}(\nabla \nu, \mathrm{A})+\mathfrak{q}^{3}(\mathrm{~A}) .
\end{aligned}
$$

Interchanging repeatedly derivatives in the first term we introduce some extra terms of the form $\mathfrak{q}^{2 m+3}(\mathrm{~A}, \mathrm{~A})$ and we get

$$
\frac{\partial}{\partial t} h_{i j}=2(-1)^{m} \overbrace{\Delta \Delta \ldots \Delta}^{m \text { times }} \nabla_{i} \nabla_{j} \mathrm{H}+\mathfrak{q}^{2 m+3}(\mathrm{~A}, \mathrm{~A})+\mathfrak{q}^{2 m+3}(\nabla \nu, \mathrm{A})+\mathfrak{q}^{3}(\mathrm{~A}),
$$


then using equation (2.4) we conclude

$$
\begin{aligned}
\frac{\partial}{\partial t} h_{i j}= & 2(-1)^{m} \overbrace{\Delta \Delta \ldots \Delta}^{m \text { times }}\left(\Delta h_{i j}-\mathrm{H} h_{i l} g^{l s} h_{s j}-|\mathrm{A}|^{2} h_{i j}\right) \\
& +\mathfrak{q}^{2 m+3}(\mathrm{~A}, \mathrm{~A})+\mathfrak{q}^{2 m+3}(\nabla \nu, \mathrm{A})+\mathfrak{q}^{3}(\mathrm{~A}) \\
= & 2(-1)^{m} \overbrace{\Delta \Delta \ldots \Delta}^{m+1 \text { times }} h_{i j}+\mathfrak{q}^{2 m+3}(\mathrm{~A}, \mathrm{~A})+\mathfrak{q}^{2 m+3}(\nabla \nu, \mathrm{A})+\mathfrak{q}^{3}(\mathrm{~A}) .
\end{aligned}
$$

Now we deal with the covariant derivatives of A.

Lemma 7.3. We have

$$
\begin{aligned}
\frac{\partial}{\partial t} \nabla^{k} h_{i j}= & 2(-1)^{m} \overbrace{\Delta \Delta \ldots \Delta}^{m+1 \text { times }} \nabla^{k} h_{i j} \\
& +\mathfrak{q}^{k+2 m+3}(\mathrm{~A}, \mathrm{~A})+\mathfrak{q}^{k+2 m+3}(\nabla \nu, \mathrm{A})+\mathfrak{q}^{k+3}(\mathrm{~A}) .
\end{aligned}
$$

Proof. With a reasoning analogous to the one of Lemma 3.5 applied to the tensor A and by the previous lemma, we have

$$
\begin{aligned}
\frac{\partial}{\partial t} \nabla^{k} h_{i j}= & \nabla^{k} \frac{\partial}{\partial t} h_{i j}+\mathfrak{p}_{k}\left(\mathrm{~A}, \mathrm{~A}, \mathrm{E}_{m}\right) \\
= & \nabla^{k} \frac{\partial}{\partial t} h_{i j}+\mathfrak{q}^{k+2 m+3}(\mathrm{~A}, \mathrm{~A})+\mathfrak{q}^{k+2 m+3}(\nabla \nu, \mathrm{A})+\mathfrak{q}^{k+3}(\mathrm{~A}, \mathrm{~A}) \\
= & 2(-1)^{m} \nabla^{k} \overbrace{\Delta \Delta \ldots \Delta}^{m+1 \text { times }} h_{i j} \\
& +\nabla^{k} \mathfrak{q}^{2 m+3}(\mathrm{~A}, \mathrm{~A})+\nabla^{k} \mathfrak{q}^{2 m+3}(\nabla \nu, \mathrm{A})+\nabla^{k} \mathfrak{q}^{3}(\mathrm{~A}) \\
& +\mathfrak{q}^{k+2 m+3}(\mathrm{~A}, \mathrm{~A})+\mathfrak{q}^{k+2 m+3}(\nabla \nu, \mathrm{A})+\mathfrak{q}^{k+3}(\mathrm{~A}, \mathrm{~A}) \\
= & 2(-1)^{m} \nabla^{k} \overbrace{\Delta \Delta \ldots \Delta}^{m+1 \text { times }} h_{i j} \\
& +\mathfrak{q}^{k+2 m+3}(\mathrm{~A}, \mathrm{~A})+\mathfrak{q}^{k+2 m+3}(\nabla \nu, \mathrm{A})+\mathfrak{q}^{k+3}(\mathrm{~A}) .
\end{aligned}
$$

Interchanging the operator $\nabla^{k}$ with the Laplacians in the first term and including the extra terms in $\mathfrak{q}^{k+2 m+3}(\mathrm{~A}, \mathrm{~A})$, we obtain

$$
\begin{aligned}
\frac{\partial}{\partial t} \nabla^{k} h_{i j}= & 2(-1)^{m} \overbrace{\Delta \Delta \ldots \Delta}^{m+1 \text { times }} \nabla^{k} h_{i j} \\
& +\mathfrak{q}^{k+2 m+3}(\mathrm{~A}, \mathrm{~A})+\mathfrak{q}^{k+2 m+3}(\nabla \nu, \mathrm{A})+\mathfrak{q}^{k+3}(\mathrm{~A}) .
\end{aligned}
$$

Proposition 7.4. The following formula holds,

$$
\begin{aligned}
\frac{\partial}{\partial t} \int_{M}\left|\nabla^{k} \mathrm{~A}\right|^{2} d \mu_{t}= & -4 \int_{M}\left|\nabla^{k+m+1} \mathrm{~A}\right|^{2} d \mu_{t} \\
& +\int_{M} \mathfrak{q}^{2(k+m+2)}(\mathrm{A}, \mathrm{A}, \mathrm{A})+\mathfrak{q}^{2(k+m+2)}(\nabla \nu, \mathrm{A}, \mathrm{A}) d \mu_{t} \\
& +\int_{M} \mathfrak{q}^{2(k+2)}(\mathrm{A}, \mathrm{A}) d \mu_{t}
\end{aligned}
$$


Proof. By the previous results we have

$$
\begin{aligned}
\frac{\partial}{\partial t}\left|\nabla^{k} \mathrm{~A}\right|^{2}= & 2 g^{i_{1} j_{1}} \ldots g^{i_{k} j_{k}} g^{i s} g^{j z} \frac{\partial}{\partial t} \nabla_{i_{1} \ldots i_{k}} h_{i j} \nabla_{j_{1} \ldots j_{k}} h_{s z} \\
& +g^{i_{1} j_{1}} \ldots \frac{\partial}{\partial t} g^{i_{l} j_{l}} \ldots g^{i_{k} j_{k}} g^{i s} g^{j z} \nabla_{i_{1} \ldots i_{k}} h_{i j} \nabla_{j_{1} \ldots j_{k}} h_{s z} \\
= & 4(-1)^{m} g^{i_{1} j_{1}} \ldots g^{i_{k} j_{k}} g^{i s} g^{j z} \overbrace{\Delta \Delta \ldots \Delta}^{m+1 \text { times }} \nabla_{i_{1} \ldots i_{k}} h_{i j} \nabla_{j_{1} \ldots j_{k}} h_{s z} \\
& +\left(\mathfrak{q}^{k+2 m+3}(\mathrm{~A}, \mathrm{~A})+\mathfrak{q}^{k+2 m+3}(\nabla \nu, \mathrm{A})+\mathfrak{q}^{k+3}(\mathrm{~A})\right) * \nabla^{k} \mathrm{~A} \\
& +2 \mathrm{E}_{m} g^{i_{1} j_{1}} \ldots h^{i_{l} j_{l}} \ldots g^{i_{k} j_{k}} g^{i s} g^{j z} \nabla_{i_{1} \ldots i_{k}} h_{i j} \nabla_{j_{1} \ldots j_{k}} h_{s z} \\
= & 4(-1)^{m} g^{i_{1} j_{1}} \ldots g^{i_{k} j_{k}} g^{i s} g^{j z} \overbrace{\Delta \Delta \ldots \Delta}^{m+1 \text { times }} \nabla_{i_{1} \ldots i_{k}} h_{i j} \nabla_{j_{1} \ldots j_{k}} h_{s z} \\
& +\mathfrak{q}^{2(k+m+2)}(\mathrm{A}, \mathrm{A}, \mathrm{A})+\mathfrak{q}^{2(k+m+2)}(\nabla \nu, \mathrm{A}, \mathrm{A})+\mathfrak{q}^{2(k+2)}(\mathrm{A}, \mathrm{A}) \\
= & 4(-1)^{m} g^{i s} g^{j z} \nabla_{i_{k+1}} \nabla^{i_{k+1}} \ldots \nabla_{i_{k+m+1}} \nabla^{i_{k+m+1}} \nabla_{i_{1} \ldots i_{k}} h_{i j} \nabla^{i_{1} \ldots i_{k}} h_{s z} \\
& +\mathfrak{q}^{2(k+m+2)}(\mathrm{A}, \mathrm{A}, \mathrm{A})+\mathfrak{q}^{2(k+m+2)}(\nabla \nu, \mathrm{A}, \mathrm{A})+\mathfrak{q}^{2(k+2)}(\mathrm{A}, \mathrm{A}) .
\end{aligned}
$$

Interchanging the covariant derivatives in the first term we introduce some extra terms of the form $\mathfrak{q}^{2(k+m+2)}(\mathrm{A}, \mathrm{A}, \mathrm{A})$, hence we get

$$
\begin{aligned}
& \frac{\partial}{\partial t} \int_{M}\left|\nabla^{k} \mathrm{~A}\right|^{2} d \mu_{t}= \\
& \quad 4(-1)^{m} \int_{M} g^{i s} g^{j z} \nabla^{i_{k+1}} \ldots \nabla^{i_{k+m+1}} \nabla_{i_{k+m+1}} \ldots \nabla_{i_{k+1}} \nabla_{i_{1} \ldots i_{k}} h_{i j} \nabla^{i_{1} \ldots i_{k}} h_{s z} d \mu_{t} \\
& \quad+\int_{M} \mathfrak{q}^{2(k+m+2)}(\mathrm{A}, \mathrm{A}, \mathrm{A})+\mathfrak{q}^{2(k+m+2)}(\nabla \nu, \mathrm{A}, \mathrm{A})+\mathfrak{q}^{2(k+2)}(\mathrm{A}, \mathrm{A}) d \mu_{t} \\
& \quad+\int_{M} \mathfrak{q}^{2(k+2)}(\mathrm{A}, \mathrm{A}) d \mu_{t}
\end{aligned}
$$

where the last integral comes from the time derivative of $\mu_{t}$.

Then, carrying the $m+1$ derivatives $\nabla^{i_{k+1}} \ldots \nabla^{i_{k+m+1}}$ on $\nabla^{i_{1} \ldots i_{k}} h_{s z}$ by means of the divergence theorem, we finally obtain the claimed result,

$$
\begin{aligned}
= & -4 \int_{M} g^{i s} g^{j z} \nabla_{i_{k+m+1}} \ldots \nabla_{i_{k+1}} \nabla_{i_{1} \ldots i_{k}} h_{i j} \nabla^{i_{k+m+1}} \ldots \nabla^{i_{k+1}} \nabla^{i_{1} \ldots i_{k}} h_{s z} d \mu_{t} \\
& +\int_{M} \mathfrak{q}^{2(k+m+2)}(\mathrm{A}, \mathrm{A}, \mathrm{A})+\mathfrak{q}^{2(k+m+2)}(\nabla \nu, \mathrm{A}, \mathrm{A})+\mathfrak{q}^{2(k+2)}(\mathrm{A}, \mathrm{A}) d \mu_{t} \\
= & -4 \int_{M}\left|\nabla^{k+m+1} \mathrm{~A}\right|^{2} d \mu_{t} \\
& +\int_{M} \mathfrak{q}^{2(k+m+2)}(\mathrm{A}, \mathrm{A}, \mathrm{A})+\mathfrak{q}^{2(k+m+2)}(\nabla \nu, \mathrm{A}, \mathrm{A})+\mathfrak{q}^{2(k+2)}(\mathrm{A}, \mathrm{A}) d \mu_{t} .
\end{aligned}
$$

The leading coefficient became -4 since we multiplied $4(-1)^{m}$ for $(-1)^{m+1}$ while doing the $m+1$ integrations by parts.

Now we analyze the terms

$$
\int_{M} \mathfrak{q}^{2(k+m+2)}(\mathrm{A}, \mathrm{A}, \mathrm{A}) d \mu_{t} \quad \text { and } \quad \int_{M} \mathfrak{q}^{2(k+m+2)}(\nabla \nu, \mathrm{A}, \mathrm{A}) d \mu_{t} .
$$

If one of the two polynomials contains a derivative $\nabla^{i} \mathrm{~A}$ or $\nabla^{i}(\nabla \nu)$ of order $i>k+m+1$, then all the other derivatives must be of order lower than $k+m$, since the rescaling order of the polynomials is $2(k+m+2)$ and the fact that there are at least three factors in every additive term. In this case, using repeatedly the divergence theorem as before, to lower such highest derivative, we get the integral of a new polynomial which does not contain derivatives of order higher than $k+m+1$. Moreover, if there is a derivative of order $k+m+1$ then the order of all the other derivatives in 
$\mathfrak{q}^{2(k+m+2)}$ must be lower or equal than $k+m$, by the same argument.

With the same reasoning, the term

$$
\int_{M} \mathfrak{q}^{2(k+2)}(\mathrm{A}, \mathrm{A}) d \mu_{t}
$$

can be transformed it in a term without derivatives of order higher or equal than $k+m+1$.

Hence, we can suppose that the last three terms in

$$
\begin{aligned}
\frac{\partial}{\partial t} \int_{M}\left|\nabla^{k} \mathrm{~A}\right|^{2} d \mu_{t}= & -4 \int_{M}\left|\nabla^{k+m+1} \mathrm{~A}\right|^{2} d \mu_{t} \\
& +\int_{M} \mathfrak{q}^{2(k+m+2)}(\mathrm{A}, \mathrm{A}, \mathrm{A})+\mathfrak{q}^{2(k+m+2)}(\nabla \nu, \mathrm{A}, \mathrm{A}) d \mu_{t} \\
& +\int_{M} \mathfrak{q}^{2(k+2)}(\mathrm{A}, \mathrm{A}) d \mu_{t}
\end{aligned}
$$

do not contain derivatives of $\mathrm{A}$ or of $\nabla \nu$ of order higher than $k+m+1$; possibly, only one derivative of order $k+m+1$ can appear.

Lemma 7.5. The following inequality holds

$$
\left|\nabla^{s} \nu\right| \leq\left|\nabla^{s-1} \mathrm{~A}\right|+\left|\mathfrak{q}^{s}(\mathrm{~A})\right|
$$

where $\mathfrak{q}^{s}(\mathrm{~A})$ does not contain derivatives of A of order higher than $s-2$.

Proof. By equations (2.5) it follows that $\nabla \nu=\mathrm{A} * \nabla \varphi$, hence

$$
\nabla^{s} \nu=\nabla^{s-1} \mathrm{~A} * \nabla \varphi+\sum_{i+j=s-2} \nabla^{i} \mathrm{~A} * \nabla^{j} \nabla^{2} \varphi
$$

and since $\nabla_{i j}^{2} \varphi=-h_{i j} \nu$, we get

$$
\begin{aligned}
\nabla^{s} \nu & =\nabla^{s-1} \mathrm{~A} * \nabla \varphi+\sum_{i+j=s-2} \nabla^{i} \mathrm{~A} * \nabla^{j}(\mathrm{~A} \nu) \\
& =\nabla^{s-1} \mathrm{~A} * \nabla \varphi+\sum_{i+j+k=s-2} \nabla^{i} \mathrm{~A} * \nabla^{j} \mathrm{~A} * \nabla^{k} \nu .
\end{aligned}
$$

Then, by an induction argument we can express $\nabla^{s} \nu$ as

$$
\nabla^{s} \nu=\nabla^{s-1} \mathrm{~A} * \nabla \varphi+\mathfrak{q}^{s}(\mathrm{~A})
$$

where $\mathfrak{q}^{s}($ A) does not contain derivatives of order higher than $s-2$.

Taking the norm of both sides we get

$$
\left|\nabla^{s} \nu\right| \leq\left|\nabla^{s-1} \mathrm{~A} * \nabla \varphi\right|+\left|\mathfrak{q}^{s}(\mathrm{~A})\right|
$$

and we conclude the proof computing

$$
\begin{aligned}
\left|\nabla^{s-1} \mathrm{~A} * \nabla \varphi\right| & =\left|\nabla_{i_{1} \ldots i_{s-1}} h_{i l} g^{l k} \frac{\partial \varphi}{\partial x_{k}}\right| \\
& =\left(\nabla_{i_{1} \ldots i_{s-1}} h_{i l} g^{l k} \frac{\partial \varphi}{\partial x_{k}} g^{i_{1} j_{1}} \ldots g^{i_{s-1} j_{s-1}} g^{i j} \nabla_{j_{1} \ldots j_{s-1}} h_{j w} g^{w z} \frac{\partial \varphi}{\partial x_{z}}\right)^{1 / 2} \\
& =\left(\nabla_{i_{1} \ldots i_{s-1}} h_{i l} g^{l k} g_{k z} g^{w z} g^{i_{1} j_{1}} \ldots g^{i_{s-1} j_{s-1}} g^{i j} \nabla_{j_{1} \ldots j_{s-1}} h_{j w}\right)^{1 / 2} \\
& =\left(\nabla_{i_{1} \ldots i_{s-1}} h_{i l} g^{l w} g^{i_{1} j_{1}} \ldots g^{i_{s-1} j_{s-1}} g^{i j} \nabla_{j_{1} \ldots j_{s-1}} h_{j w}\right)^{1 / 2} \\
& =\left|\nabla^{s-1} \mathrm{~A}\right| .
\end{aligned}
$$

Taking the absolute values inside the integrals and using this lemma to substitute every derivative of $\nu$ in (7.1), we obtain

$$
\frac{\partial}{\partial t} \int_{M}\left|\nabla^{k} \mathrm{~A}\right|^{2} d \mu_{t} \leq-4 \int_{M}\left|\nabla^{k+m+1} \mathrm{~A}\right|^{2} d \mu_{t}+\int_{M}\left|\mathfrak{q}^{2(k+m+2)}(\mathrm{A})\right|+\left|\mathfrak{q}^{2(k+2)}(\mathrm{A})\right| d \mu_{t}
$$


where, as before, the two polynomials do not contain derivatives of $\mathrm{A}$ of order higher than $k+$ $m+1$; possibly, only one derivative of order $k+m+1$ can appear in every multiplicative term of $\mathfrak{q}^{2(k+m+2)}(\mathrm{A})$.

Before going on, we remark that the $*$ product of tensors satisfies the following metric property,

$$
|T * S| \leq|T| \cdot|S| \text {. }
$$

This can be easily seen choosing an orthonormal basis at a point of $M$, in such coordinates we have

$$
\begin{aligned}
|T * S|^{2} & =\sum_{\substack{\text { free } \\
\text { indices }}}\left(\sum_{\substack{\text { contracted } \\
\text { indices }}} T_{i_{1} \ldots i_{k}} S_{j_{1} \ldots j_{l}}\right)^{2} \\
& \leq \sum_{\substack{\text { free } \\
\text { indices }}}\left(\sum_{\substack{\text { contracted } \\
\text { indices }}} T_{i_{1} \ldots i_{k}}^{2}\right)\left(\sum_{\substack{\text { contracted } \\
\text { indices }}} S_{j_{1} \ldots j_{l}}^{2}\right) \\
& \leq\left(\sum_{\substack{\text { free } \\
\text { indices }}} \sum_{\substack{\text { contracted } \\
\text { indices }}} T_{i_{1} \ldots i_{k}}^{2}\right)\left(\sum_{\substack{\text { free } \\
\text { indices }}} \sum_{\substack{\text { contracted } \\
\text { indices }}} S_{j_{1} \ldots j_{l}}^{2}\right) \\
& =|T|^{2} \cdot|S|^{2} .
\end{aligned}
$$

Now by definition we have

$$
\mathfrak{q}^{2(k+m+2)}(\mathrm{A})=\sum_{j} \stackrel{N_{j}}{\circledast} \nabla_{l=1}^{c_{j l}} \mathrm{~A}
$$

with

$$
\sum_{l=1}^{N_{j}}\left(c_{j l}+1\right)=2(k+m+2)
$$

for every $j$, hence

$$
\left|\mathfrak{q}^{2(k+m+2)}(\mathrm{A})\right| \leq \sum_{j} \prod_{l=1}^{N_{j}}\left|\nabla^{c_{j l}} \mathrm{~A}\right|
$$

by (7.2). Setting

$$
Q_{j}=\prod_{l=1}^{N_{j}}\left|\nabla^{c_{j l}} \mathrm{~A}\right|
$$

we clearly obtain

$$
\int_{M}\left|\mathfrak{q}^{2(k+m+2)}(\mathrm{A})\right| d \mu_{t} \leq \sum_{j} \int_{M} Q_{j} d \mu_{t}
$$

If $Q_{j}$ contains a derivative of A of order $k+m+1$, we have seen that all the others have order lower or equal than $k+m$, then collecting derivatives of the same order, $Q_{j}$ can be estimated as follows

$$
Q_{j} \leq\left|\nabla^{k+m+1} \mathrm{~A}\right| \cdot \prod_{i=0}^{k+m}\left|\nabla^{i} \mathrm{~A}\right|^{\alpha_{j i}}
$$

for some $\alpha_{j i}$ satisfying the rescaling condition

$$
(k+m+2)+\sum_{i=0}^{k+m}(i+1) \alpha_{j i}=2(k+m+2) .
$$

Hence, using Young inequality, for every $\varepsilon_{j}>0$ we have

$$
\begin{aligned}
\int_{M} Q_{j} d \mu_{t} & \leq \varepsilon_{j} \int_{M}\left|\nabla^{k+m+1} \mathrm{~A}\right|^{2} d \mu_{t}+\frac{1}{4 \varepsilon_{j}} \int_{M} \prod_{i=0}^{k+m}\left|\nabla^{i} \mathrm{~A}\right|^{2 \alpha_{j i}} d \mu_{t} \\
& =\varepsilon_{j} \int_{M}\left|\nabla^{k+m+1} \mathrm{~A}\right|^{2} d \mu_{t}+\int_{M}\left|\mathfrak{q}^{2(k+m+2)}(\mathrm{A})\right| d \mu_{t}
\end{aligned}
$$


where we put in evidence the fact that the last term satisfies again the rescaling condition and no more contains the derivative $\nabla^{k+m+1} \mathrm{~A}$.

Collecting all together such "bad" terms, and choosing suitable $\varepsilon_{j}>0$ such that their total sum is less than one, we obtain

$$
\frac{\partial}{\partial t} \int_{M}\left|\nabla^{k} \mathrm{~A}\right|^{2} d \mu_{t} \leq-3 \int_{M}\left|\nabla^{k+m+1} \mathrm{~A}\right|^{2} d \mu_{t}+\int_{M}\left|\mathfrak{q}^{2(k+m+2)}(\mathrm{A})\right|+\int_{M}\left|\mathfrak{q}^{2(k+2)}(\mathrm{A})\right| d \mu_{t}
$$

where now in the last two terms all the derivatives of A have order lower than $k+m+1$. We are then ready to estimate them via interpolation inequalities.

As before,

$$
\left|\mathfrak{q}^{2(k+m+2)}(\mathrm{A})\right| \leq \sum_{j} Q_{j}
$$

and after collecting derivatives of the same order in $Q_{j}$,

$$
Q_{j}=\prod_{i=0}^{k+m}\left|\nabla^{i} \mathrm{~A}\right|^{\alpha_{j i}} \quad \text { with } \quad \sum_{i+1}^{k+m} \alpha_{j i}(i+1)=2(k+m+2) .
$$

Then,

$$
\begin{aligned}
\int_{M} Q_{j} d \mu_{t} & =\int_{M} \prod_{i=0}^{k+m}\left|\nabla^{i} \mathrm{~A}\right|^{\alpha_{j i}} d \mu_{t} \\
& \leq \prod_{i=0}^{k+m}\left(\int_{M}\left|\nabla^{i} \mathrm{~A}\right|^{\alpha_{j i} \gamma_{i}} d \mu_{t}\right)^{\frac{1}{\gamma_{i}}} \\
& =\prod_{i=0}^{k+m}\left\|\nabla^{i} \mathrm{~A}\right\|_{L^{\alpha_{j i} \gamma_{i}}\left(\mu_{t}\right)}^{\alpha_{i j}}
\end{aligned}
$$

where the $\gamma_{i}$ are arbitrary positive values such that $\sum 1 / \gamma_{i}=1$.

We apply interpolation inequalities: if in (6.7) we take $q=2, r=n+1, s=k+m+1, j=i$ and $T=\mathrm{A}$ we get

$$
\left\|\nabla^{i} \mathrm{~A}\right\|_{L^{p_{i}\left(\mu_{t}\right)}} \leq C\|\mathrm{~A}\|_{W^{2, k+m+1}\left(\mu_{t}\right)}^{a}\|\mathrm{~A}\|_{L^{n+1}\left(\mu_{t}\right)}^{1-a}
$$

with

$$
a=\frac{\frac{1}{p_{i}}-\frac{i}{n}-\frac{1}{n+1}}{\frac{1}{2}-\frac{k+m+1}{n}-\frac{1}{n+1}} \in\left[\frac{i}{k+m+1}, 1\right]
$$

and $p_{i}>1$.

Now, since the volumes of $M_{t}$ and $\|\mathrm{A}\|_{L^{n+1}\left(\mu_{t}\right)}$ are uniformly bounded in time, also $\|\mathrm{A}\|_{L^{2}\left(\mu_{t}\right)}$ is uniformly bounded and using the universal inequalities (6.6) with $p=q=r=2$ we have

$$
\begin{aligned}
\|\mathrm{A}\|_{W^{2, k+m+1}\left(\mu_{t}\right)} & \leq \sum_{s=0}^{k+m+1} C\left\|\nabla^{k+m+1} \mathrm{~A}\right\|_{L^{2}\left(\mu_{t}\right)}^{\frac{s}{k+m+1}} \\
& \leq \sum_{s=0}^{k+m+1}\left\|\nabla^{k+m+1} \mathrm{~A}\right\|_{L^{2}\left(\mu_{t}\right)}+C \\
& \leq B\left\|\nabla^{k+m+1} \mathrm{~A}\right\|_{L^{2}\left(\mu_{t}\right)}+C,
\end{aligned}
$$

where we applied Young inequality.

Hence, we conclude that we have constants $B, C$ independent of $t$ such that

$$
\left\|\nabla^{i} \mathrm{~A}\right\|_{L^{p_{i}\left(\mu_{t}\right)}} \leq\left(B\left\|\nabla^{k+m+1} \mathrm{~A}\right\|_{L^{2}\left(\mu_{t}\right)}+C\right)^{a}
$$

for $a$ as in (7.3) and $p_{i}>1$. 
Choosing $\gamma_{i}=0$ if $\alpha_{j i}=0$ and $\gamma_{i}=\frac{2(k+m+2)}{\alpha_{j i}(i+1)}$ otherwise, we have clearly

$$
\sum_{i=0}^{k+m} \frac{1}{\gamma_{i}}=\sum_{i=0}^{k+m} \frac{\alpha_{j i}(i+1)}{2(k+m+2)}=1
$$

by the rescaling condition on the $\alpha_{j i}$.

We claim that for every $i \in\{0, \ldots, k+m\}$, the product $p_{i}=\alpha_{j i} \gamma_{i}$ satisfies the condition (7.3).

By definition, $p_{i}=\frac{2(k+m+2)}{i+1}$, hence we must check that the following inequality holds

$$
\frac{i}{k+m+1} \leq \frac{\frac{i+1}{2(k+m+2)}-\frac{i}{n}-\frac{1}{n+1}}{\frac{1}{2}-\frac{k+m+1}{n}-\frac{1}{n+1}} \leq 1
$$

for every $i \in\{0, \ldots, k+m\}$. Since every term is an affine function of $i$, the claim follows if we show that the inequality holds for $i=0$ and $i=k+m+1$.

If $i=0$ we have to prove that

$$
0 \leq \frac{\frac{1}{2(k+m+2)}-\frac{1}{n+1}}{\frac{1}{2}-\frac{k+m+1}{n}-\frac{1}{n+1}} \leq 1,
$$

that is, since the denominator of the fraction is negative (as $2 m \geq n+1)$,

$$
\frac{1}{2}-\frac{k+m+1}{n}-\frac{1}{n+1} \leq \frac{1}{2(k+m+2)}-\frac{1}{n+1} \leq 0 .
$$

The right inequality is clearly true, again since $2 m \geq n+1$, the left one becomes

$$
\frac{k+m+1}{2(k+m+2)}=\frac{1}{2}-\frac{1}{2(k+m+2)} \leq \frac{k+m+1}{n}
$$

which is true as $2(k+m+2) \geq n$.

When $i=k+m+1$ the fraction is equal to 1 , hence the inequality obviously holds.

Then, the exponents $p_{i}=\alpha_{j i} \gamma_{i}$ are allowed in inequality (7.4) and we get

$$
\left\|\nabla^{i} \mathrm{~A}\right\|_{L^{\alpha} \gamma_{j i}\left(\mu_{t}\right)} \leq\left(B\left\|\nabla^{k+m+1} \mathrm{~A}\right\|_{L^{2}\left(\mu_{t}\right)}+C\right)^{a_{j i}}
$$

where $a_{j i}$ is the relative value we obtain from (7.3).

Hence,

$$
\begin{aligned}
\int_{M} Q_{j} d \mu_{t} & \leq \prod_{i=0}^{k+m}\left\|\nabla^{i} \mathrm{~A}\right\|_{L^{\alpha j i} \gamma_{i}\left(\mu_{t}\right)}^{\alpha_{i j}} \\
& \leq \prod_{i=0}^{k+m}\left(B\left\|\nabla^{k+m+1} \mathrm{~A}\right\|_{L^{2}\left(\mu_{t}\right)}+C\right)^{a_{j i} \alpha_{j i}} \\
& \leq\left(B\left\|\nabla^{k+m+1} \mathrm{~A}\right\|_{L^{2}\left(\mu_{t}\right)}+C\right)^{\sum_{i=0}^{k+m} a_{j i} \alpha_{j i}}
\end{aligned}
$$

where the constants $B$ and $C$ are independent of $t$ and

$$
a_{j i}=\frac{\frac{1}{\alpha_{j i} \gamma_{i}}-\frac{i}{n}-\frac{1}{n+1}}{\frac{1}{2}-\frac{k+m+1}{n}-\frac{1}{n+1}} .
$$

Multiplying this relation by $\alpha_{j i}$ and summing on $i$ from 0 to $k+m$ we get

$$
\begin{aligned}
\sum_{i=0}^{k+m} \alpha_{j i} a_{j i} & =\sum_{i=0}^{k+m} \frac{\frac{1}{\gamma_{i}}-\frac{i \alpha_{j i}}{n}-\frac{\alpha_{j i}}{n+1}}{\frac{1}{2}-\frac{k+m+1}{n}-\frac{1}{n+1}} \\
& =\frac{1-\sum_{i=0}^{k+m}\left(\frac{i \alpha_{j i}}{n}+\frac{\alpha_{j i}}{n+1}\right)}{\frac{1}{2}-\frac{k+m+1}{n}-\frac{1}{n+1}} \\
& =\frac{1-\sum_{i=0}^{k+m} \frac{\alpha_{j i}(i+1)}{n}-\sum_{i=0}^{k+m} \alpha_{j i}\left(\frac{1}{n+1}-\frac{1}{n}\right)}{\frac{1}{2}-\frac{k+m+1}{n}-\frac{1}{n+1}}
\end{aligned}
$$


recalling that $\sum_{i=0}^{k+m} \alpha_{j i}(i+1)=2(k+m+2)$ we continue,

$$
\begin{aligned}
& =\frac{1-2 \frac{k+m+2}{n}+\sum_{i=0}^{k+m} \frac{\alpha_{j i}}{n(n+1)}}{\frac{1}{2}-\frac{k+m+1}{n}-\frac{1}{n+1}} \\
& =\frac{1-2 \frac{k+m+1}{n}-\frac{2}{n}+\sum_{i=0}^{k+m} \frac{\alpha_{j i}}{n(n+1)}}{\frac{1}{2}-\frac{k+m+1}{n}-\frac{1}{n+1}} .
\end{aligned}
$$

Now, the denominator is negative and clearly

$$
\sum_{i=0}^{k+m} \alpha_{j i} \geq \sum_{i=0}^{k+m} \frac{\alpha_{j i}(i+1)}{k+m+1}=2 \frac{k+m+2}{k+m+1},
$$

so we obtain

$$
\begin{aligned}
\sum_{i=0}^{k+m} \alpha_{j i} a_{j i} & \leq \frac{1-2 \frac{k+m+1}{n}-\frac{2}{n}+2 \frac{k+m+2}{k+m+1} \frac{1}{n(n+1)}}{\frac{1}{2}-\frac{k+m+1}{n}-\frac{1}{n+1}} \\
& =\frac{1-2 \frac{k+m+1}{n}-\frac{2}{n}+\frac{2}{n(n+1)}+\frac{2}{k+m+1} \frac{1}{n(n+1)}}{\frac{1}{2}-\frac{k+m+1}{n}-\frac{1}{n+1}} \\
& =\frac{1-2 \frac{k+m+1}{n}-\frac{2}{n+1}+\frac{2}{k+m+1} \frac{1}{n(n+1)}}{\frac{1}{2}-\frac{k+m+1}{n}-\frac{1}{n+1}} \\
& =2-\frac{2}{\frac{k+m+1}{n}+\frac{1}{n(n+1)}} \frac{1}{n+1}-\frac{1}{2} \\
& =2-\frac{4}{(k+m+1)[2(k+m+1)(n+1)-n(n-1)]}<2 .
\end{aligned}
$$

Hence, we finally get

$$
\int_{M} Q_{j} d \mu_{t} \leq\left(B \int_{M}\left|\nabla^{k+m+1} \mathrm{~A}\right|^{2} d \mu_{t}+C\right)^{1-\delta}
$$

for a positive $\delta$ and using again Young inequality, we have

$$
\int_{M} Q_{j} d \mu_{t} \leq \varepsilon_{j} \int_{M}\left|\nabla^{k+m+1} \mathrm{~A}\right|^{2} d \mu_{t}+C
$$

for arbitrarily small $\varepsilon_{j}$. Repeating this argument for all the $Q_{j}$ and choosing suitable $\varepsilon_{j}$ whose sum is less than one, we conclude that

$$
\frac{d}{d t} \int_{M}\left|\nabla^{k} \mathrm{~A}\right|^{2} \mu_{t} \leq-2 \int_{M}\left|\nabla^{k+m+1} \mathrm{~A}\right|^{2} \mu_{t}+C+\int_{M}\left|\mathfrak{q}^{2(k+2)}(\mathrm{A})\right| d \mu_{t}
$$

with a constant $C$ independent of time.

The last term can be treated in the same way. It can be estimated by the sum of the multiplicative terms $Q_{j}$ and collecting derivatives of the same order as before, we have

$$
Q_{j} \leq \prod_{i=0}^{k+m}\left|\nabla^{i} \mathrm{~A}\right|^{\beta_{j i}} \quad \text { with } \quad \sum_{i=0}^{k+m} \beta_{j i}(i+1)=2 k+4
$$

In this case the coefficients $\gamma_{i}$, when $\beta_{j i} \neq 0$, are given by $\gamma_{i}=\frac{2(k+2)}{\alpha_{j i}(i+1)}$, hence

$$
\sum_{i=0}^{k+m} \frac{1}{\gamma_{i}}=\sum_{i=0}^{k+m} \frac{\alpha_{j i}(i+1)}{2(k+2)}=1
$$

by the rescaling condition.

With an analogous control, one can see that the conditions on the exponent $p_{i}$ are satisfied. It 
lasts to compute

$$
\begin{aligned}
\sum_{i=0}^{k+m} \beta_{j i} a_{j i} & =\sum_{i=0}^{k+m} \frac{\frac{1}{\gamma_{i}}-\frac{i \beta_{j i}}{n}-\frac{\beta_{j i}}{n+1}}{\frac{1}{2}-\frac{k+m+1}{n}-\frac{1}{n+1}} \\
& =\frac{1-\sum_{i=0}^{k+m}\left(\frac{i \beta_{j i}}{n}+\frac{\beta_{j i}}{n+1}\right)}{\frac{1}{2}-\frac{k+m+1}{n}-\frac{1}{n+1}} \\
& =\frac{1-\sum_{i=0}^{k+m} \frac{\beta_{j i}(i+1)}{n}+\sum_{i=0}^{k+m} \frac{\beta_{j i}}{n(n+1)}}{\frac{1}{2}-\frac{k+m+1}{n}-\frac{1}{n+1}} \\
& =\frac{1-\frac{2 k+4}{n}+\sum_{i=0}^{k+m} \frac{\beta_{j i}}{n(n+1)}}{\frac{1}{2}-\frac{k+m+1}{n}-\frac{1}{n+1}} .
\end{aligned}
$$

As the denominator is negative and

$$
\sum_{i=0}^{k+m} \beta_{j i} \geq \sum_{i=0}^{k+m} \frac{\beta_{j i}(i+1)}{k+m+1}=\frac{2 k+4}{k+m+1},
$$

we obtain

$$
\begin{aligned}
\sum_{i=0}^{k+m} \beta_{j i} a_{j i} & \leq \frac{1-\frac{2 k+4}{n}+\sum_{i=0}^{k+m} \frac{\beta_{j i}(i+1)}{k+m+1} \frac{1}{n(n+1)}}{\frac{1}{2}-\frac{k+m+1}{n}-\frac{1}{n+1}} \\
& =\frac{1-\frac{2 k+4}{n}+\frac{2 k+4}{k+m+1} \frac{1}{n(n+1)}}{\frac{1}{2}-\frac{k+m+1}{n}-\frac{1}{n+1}}<2,
\end{aligned}
$$

since this last inequality is equivalent to

$$
1-\frac{2 k+4}{n}+\frac{2 k+4}{k+m+1} \frac{1}{n(n+1)}>1-\frac{2(k+m+1)}{n}-\frac{2}{n+1}
$$

and simplifying, to

$$
\frac{2 k+4}{k+m+1} \frac{1}{n(n+1)}>-\frac{2(m-1)}{n}-\frac{2}{n+1}
$$

which is obviously true.

Concluding as before we finally get

$$
\frac{d}{d t} \int_{M}\left|\nabla^{k} \mathrm{~A}\right|^{2} \mu_{t} \leq-\int_{M}\left|\nabla^{k+m+1} \mathrm{~A}\right|^{2} \mu_{t}+C
$$

for a constant $C$ independent of time.

By (5.2) and Young inequality, we have

$$
\begin{aligned}
\int_{M}\left|\nabla^{k} \mathrm{~A}\right|^{2} \mu_{t}+C & \leq B\left\|\nabla^{k+m+1} \mathrm{~A}\right\|_{L^{2}\left(\mu_{t}\right)}^{\frac{k}{k+m+1}}\|\mathrm{~A}\|_{L^{2}\left(\mu_{t}\right)}^{\frac{m+1}{k+m+1}}+C \\
& \leq B\left\|\nabla^{k+m+1} \mathrm{~A}\right\|_{L^{2}\left(\mu_{t}\right)}^{\frac{k}{k+m+1}}+C \\
& \leq \frac{1}{2} \int_{M}\left|\nabla^{k+m+1} \mathrm{~A}\right|^{2} \mu_{t}+C
\end{aligned}
$$

again with a uniform constant. Combining this inequality with (7.5), we obtain

$$
\frac{d}{d t} \int_{M}\left|\nabla^{k} \mathrm{~A}\right|^{2} \mu_{t} \leq-\frac{1}{2} \int_{M}\left|\nabla^{k} \mathrm{~A}\right|^{2} \mu_{t}+C
$$

and a simple ODE's argument proves that there exists constants $C_{k}$ independent of time such that

$$
\int_{M}\left|\nabla^{k} \mathrm{~A}\right|^{2} d \mu_{t} \leq C_{k}
$$


To pass from $W^{2, p}\left(\mu_{t}\right)$ to pointwise estimates, first we notice that being all the derivatives of A bounded in $L^{2}\left(\mu_{t}\right)$, by inequalities (6.2), for every $p \geq 1$ and $k \in \mathbb{N}$ we have constants $C_{k, p}$ such that

$$
\int_{M}\left|\nabla^{k} \mathrm{~A}\right|^{p} d \mu_{t} \leq C_{k, p}
$$

Then choosing a $p>n$, we apply inequalities (6.3) to every $\nabla^{k} \mathrm{~A}$ to conclude that for every $k \in \mathbb{N}$ we have constants $C_{k}$, independent of $t$, such that

$$
\max _{M_{t}}\left|\nabla^{k} \mathrm{~A}\right| \leq C_{k}
$$

Looking back at the way we obtained them, we can see that the constants $C_{k}$ depend only on the dimension $n$, the differentiation order $k$ and the initial hypersurface $\varphi_{0}$.

Following Huisken [21, Sec. 8 and Kuwert and Schätzle [26], Sec. 4, these estimates imply the smoothness of the map $\varphi(p, t)$.

Since $\nabla^{k} A$ are uniformly bounded in time, supposing that $[0, T)$ is the maximal interval of existence of the flow, we have

$$
|\varphi(p, t)-\varphi(p, s)| \leq \int_{s}^{t}\left|\mathrm{E}_{m}\left(\varphi_{\xi}\right)(p)\right| d \xi \leq C(t-s)
$$

for every $0 \leq s \leq t<T$, then $\varphi_{t}$ uniformly converge to a continuous limit $\varphi_{T}$ as $t \rightarrow T$.

We recall Lemma 8.2 in [21] (Lemma 14.2 in [20]).

Lemma 7.6. Let $g_{i j}$ a time-dependent metric on a compact manifold $M$ for $0 \leq t<T \leq+\infty$. Suppose that

$$
\int_{0}^{T} \max _{M_{t}}\left|\frac{\partial}{\partial t} g_{i j}\right| d t \leq C
$$

Then the metrics $g_{i j}(t)$ are all equivalent, and they converge as $t \rightarrow T$ uniformly to a positive definite metric tensor $g_{i j}(T)$ which is continuous and also equivalent.

In our situation, if $T<+\infty$, the hypotheses of this lemma are clearly satisfied, hence $\varphi(\cdot, T)$ represents a hypersurface. Moreover, it also follows that there exists a positive constant $C$ depending only on $n$ and $\varphi_{0}$ such that for every $0 \leq t<T$ we have

$$
\frac{1}{C} \leq g_{i j}(t) \leq C
$$

Since

by (7.6), for every $k \in \mathbb{N}$ we have

$$
\frac{\partial}{\partial t} g_{i j}=-2 \mathrm{E}_{m} h_{i j}
$$

$$
\left\|\nabla^{k} \frac{\partial}{\partial t} g_{i j}\right\|_{L^{\infty}(\mu)} \leq C_{k},
$$

analogously, as the time derivative of the Christoffel symbols is given by

$$
\frac{\partial}{\partial t} \Gamma_{j k}^{i}=\nabla \mathrm{E}_{m} * \mathrm{~A}+\mathrm{E}_{m} * \nabla \mathrm{A}
$$

it follows that

$$
\left\|\nabla^{k} \frac{\partial}{\partial t} \Gamma_{j k}^{i}\right\|_{L^{\infty}(\mu)} \leq C_{k} .
$$

for every $k \in \mathbb{N}$.

With an induction argument, we can prove the following formula (where we avoid to indicate the indices) relating the iterated covariant and coordinate derivatives of a tensor $T$,

$$
\nabla^{m} T=\partial^{m} T+\sum_{i=1}^{m} \sum_{j_{1}+\cdots+j_{i}+k \leq m-1} \partial^{j_{1}} \Gamma \ldots \partial^{j_{i}} \Gamma \partial^{k} T .
$$


By this formula and induction, it follows that

$$
\left\|\partial^{k} \Gamma_{j l}^{i}\right\|_{L^{\infty}(\mu)}, \quad\left\|\partial^{k} \frac{\partial}{\partial t} \Gamma_{j l}^{i}\right\|_{L^{\infty}(\mu)} \leq C_{k},
$$

for every $t \in[0, T)$.

Applying again formula $(7.7)$ to $T=\nabla^{s} \mathrm{~A}$ we see that

$$
\partial^{k} \nabla^{s} \mathrm{~A}-\nabla^{k+s} \mathrm{~A}=\sum_{i=1}^{k} \sum_{j_{1}+\cdots+j_{i}+l \leq k-1} \partial^{j_{1}} \Gamma \ldots \partial^{j_{i}} \Gamma \partial^{l} \nabla^{s} \mathrm{~A},
$$

and by induction and estimates (7.6) we obtain

$$
\left\|\partial^{k} \nabla^{s} \mathrm{~A}\right\|_{L^{\infty}(\mu)} \leq C_{k, s}
$$

for every $k, s \in \mathbb{N}$.

Since we already know that $|\varphi|$ is bounded and $|\partial \varphi|=1$, by the Gauss-Weingarten relations (2.5)

$$
\partial^{2} \varphi=\Gamma \partial \varphi+\mathrm{A} \nu, \quad \partial \nu=\mathrm{A} * \partial \varphi
$$

and the previous estimates, we can conclude that

$$
\left\|\partial^{k} \varphi\right\|_{L^{\infty}(\mu)} \leq C_{k}
$$

for every $k \in \mathbb{N}$ and $t \in[0, T)$.

The regularity of the time derivatives also follows by these estimates and the evolution equation.

Hence, the convergence $\varphi_{t} \rightarrow \varphi_{T}$, when $t \rightarrow T$, is in the $C^{\infty}$ topology and $M_{T}$ is smooth. Then, using Theorem 4.1 to restart the flow with $\varphi_{T}$ as initial hypersurface, we get a contradiction with the fact that $[0, T)$ is the maximal interval of existence.

Remark 7.7. Though this argument shows that the solution is classical, we cannot conclude that the estimates on the parametrization hold uniformly for every $t \in[0,+\infty)$ which is instead the case for the estimates (7.6) on the curvature.

Theorem 7.8. If $m>\left[\frac{n}{2}\right]$, for any smooth hypersurface immersion $\varphi_{0}: M \rightarrow \mathbb{R}^{n+1}$ there exists a unique smooth solution to the problem

$$
\frac{\partial \varphi}{\partial t}(p, t)=-\mathrm{E}_{m}\left(\varphi_{t}\right)(p) \nu(p, t),
$$

that is, the gradient flow associated to the functional

$$
\mathcal{F}_{m}(\varphi)=\int_{M} 1+\left|\nabla^{m} \nu\right|^{2} d \mu,
$$

defined for every $t \in[0,+\infty)$ and taking $\varphi_{0}$ as its initial value.

Moreover, such solution satisfies

$$
\max _{M_{t}}\left|\nabla^{k} \mathrm{~A}\right| \leq C_{k}
$$

for constants $C_{k}$ depending only on $n, k$ and $\varphi_{0}$.

\section{Convergence}

Let us consider the function $\sigma:[0,+\infty) \rightarrow \mathbb{R}$,

$$
\sigma(t)=\int_{M}\left[\mathrm{E}_{m}\left(\varphi_{t}\right)\right]^{2} d \mu_{t} \geq 0 .
$$

Clearly we have

$$
\frac{d}{d t} \mathcal{F}_{m}\left(\varphi_{t}\right)=-\int_{M}\left[\mathrm{E}_{m}\left(\varphi_{t}\right)\right]^{2} d \mu_{t}=-\sigma(t),
$$

and integrating both sides in $t$ on $[0,+\infty)$ we get

$$
\int_{0}^{+\infty} \sigma(t) d t=\mathcal{F}_{m}\left(\varphi_{0}\right)-\mathcal{F}_{m}\left(\varphi_{t}\right) \leq \mathcal{F}_{m}\left(\varphi_{0}\right) .
$$


Moreover,

$$
\left|\frac{d}{d t} \sigma(t)\right|=\int_{M}\left|2 \frac{\partial \mathrm{E}_{m}\left(\varphi_{t}\right)}{\partial t} \mathrm{E}_{m}\left(\varphi_{t}\right)-\mathrm{H}\left[\mathrm{E}_{m}\left(\varphi_{t}\right)\right]^{3}\right| d \mu_{t} \leq C
$$

by the bounds $(7.6)$. Then the function $\sigma$, being Lipschitz and integrable on $[0,+\infty)$, converges to zero at $+\infty$. This means that every $C^{\infty}$ limit hypersurface of the flow $\psi: M \rightarrow \mathbb{R}^{n+1}$ satisfies $\mathrm{E}_{m}(\psi)=0$, i. e., it is a critical point of $\mathcal{F}_{m}$. 27.

To find limit hypersurfaces, we need the following compactness result of Langer and Delladio 13 ,

Theorem 8.1. Let be given a family $\left(M, g_{i}\right)$ of closed, oriented, $n$-dimensional hypersurfaces, isometrically immersed in $\mathbb{R}^{n+1}$ via the maps $\varphi_{i}: M \rightarrow \mathbb{R}^{n+1}$, let $\mu_{i}$ the associated measures on $M$ and $\operatorname{Bar}_{i}$ the center of gravity of $\varphi_{i}$, that is,

$$
\operatorname{Bar}_{i}=\int_{M} \varphi_{i} d \mu_{i}
$$

Let $h$ be any metric tensor on $M$, if for some exponent $p>n$ and $C>0$ we have

$$
\int_{M} 1+|\mathrm{A}|^{p} d \mu_{i}+\left|\operatorname{Bar}_{i}\right| \leq C<+\infty
$$

then there exist a subsequence of $\left\{\varphi_{i}\right\}$ (not relabeled) and diffeomorphisms $\sigma_{i}: M \rightarrow M$ such that, $\left\{\varphi_{i} \circ \sigma_{i}\right\}$ converges in the $H^{2, p}$ weak topology of maps from $(M, h) \rightarrow \mathbb{R}^{n+1}$ to an immersion $\varphi: M \rightarrow \mathbb{R}^{n+1}$.

Translating the hypersurfaces $\varphi_{t}: M \rightarrow \mathbb{R}$ in order to have $\operatorname{Bar}_{t}=0 \in \mathbb{R}^{n+1}$, we are in the above hypotheses. Hence, we can extract a subsequence of smooth hypersurfaces $\varphi_{i}=\varphi_{t_{i}}$ and diffeomorphisms $\sigma_{i}: M \rightarrow M$ such that, for a fixed metric $h$ on $M$, the sequence $\left\{\varphi_{i} \circ \sigma_{i}\right\}$ converges in the $H^{2, p}$ weak topology to an immersion $\psi: M \rightarrow \mathbb{R}^{n+1}$.

With the arguments of the proof of Theorem 8.1 in 13, 27. and keeping into account that in our case we have also the estimates (7.6), it is possible to conclude that actually the convergence is in the $C^{\infty}$ topology and the limit hypersurface is smooth (see also [22], Prop. 3.4).

Theorem 8.2. The family of smooth hypersurfaces $\varphi_{0}: M \rightarrow \mathbb{R}^{n+1}$, immersed in $\mathbb{R}^{n+1}$, evolving by the gradient flow for the functional

$$
\mathcal{F}_{m}(\varphi)=\int_{M} 1+\left|\nabla^{m} \nu\right|^{2} d \mu
$$

when $m>\left[\frac{n}{2}\right]$, up to reparametrizations and translations, is compact in the $C^{\infty}$ topology of maps. Moreover, every limit point for $t \rightarrow+\infty$ is a $C^{\infty}$ critical hypersurface of the functional $\mathcal{F}_{m}$.

\section{Some Remarks and Open Problems}

9.1. Other Ambient Spaces. A natural extension would be to consider an ambient spaces different by $\mathbb{R}^{n+1}$ and a codimension $s$ greater than one, that is, a general Riemannian manifold $(N, h)$ of dimension $n+s$ (notice that Polden's Theorem 4.1 about small time existence of the flow already deals with hypersurfaces in a general target manifold). In this context a functional which could be considered is

$$
\mathcal{F}_{m}(\varphi)=\int_{M} 1+\left|\nabla^{m} \omega\right|^{2} d \mu
$$

where $\omega=\nu_{1} \wedge \cdots \wedge \nu_{s}$ is a $s$-vector obtained by a local orthonormal basis of the normal space to the $n$-dimensional immersed submanifold $\varphi: M \rightarrow N^{n+s}$.

In 26] Kuwert and Schätzle announce a forthcoming paper with the extension of Polden's results to space curves. 
9.2. Other Functionals. It would be very interesting to study the flows in the "critical" case $2 m=n$, where our proof fails since we are no more able to bound the constants independently of time. Notice that the well known Willmore functional (see [26, 34, 39])

$$
\mathcal{W}(\varphi)=\int_{M}|\mathrm{~A}|^{2} d \mu
$$

falls exactly in this case if we add the area term, since $|\mathrm{A}|^{2}$ is equal to $|\nabla \nu|^{2}$.

To the author knowledge, up to now nor there is a proof of regularity of the flow, neither an example showing the development of a singularity. A first step in this research was recently done by Kuwert and Schätzle 26].

When $2 m<n$ we do not expect regularity of the flow by the gradient of $\mathcal{F}_{m}$ since, by analogy with the previous discussion about the regularity of varifolds, the curvature term should not be sufficient to give regularity and dumb-bell like separation phenomena should appear during the flow of certain hypersurfaces. It should also be noticed that in this and in the critical case, the $n$-dimensional unit sphere in $\mathbb{R}^{n+1}$ collapses in finite time.

Moreover, one can consider also "non-quadratic" functionals, for instance,

$$
\mathcal{F}_{m, p}(\varphi)=\int_{M} 1+\left|\nabla^{m} \nu\right|^{p} d \mu \quad \text { when } m p>n
$$

(following the analogy with the Sobolev spaces), in particular,

$$
\mathcal{F}_{1, p}(\varphi)=\int_{M} 1+|\mathrm{A}|^{p} d \mu \quad \text { for } p>n
$$

which would give rise to a flow of order lower than the one of $\mathcal{F}_{m}$ when $n>1$.

In the same spirit another interesting functional is

$$
\mathcal{H}_{p}(\varphi)=\int_{M} 1+|\mathrm{H}|^{p} d \mu \quad \text { for } p>n .
$$

In these cases the smoothness of the associated flows is an open problem.

9.3. Smoothing Terms. From our analysis, it easily follows that for every positive constants $\alpha$ and $\beta$ also the gradient flow of the functional

$$
\mathcal{F}_{m}^{\alpha \beta}(\varphi)=\int_{M} \alpha+\beta\left|\nabla^{m} \nu\right|^{2} d \mu
$$

exists and it is smooth for every positive time.

Moreover, if we consider a general positive geometric functional

$$
\mathcal{G}(\varphi)=\int_{M} f\left(\varphi, g, \mathrm{~A}, \nu, \ldots, \nabla^{s} \mathrm{~A}, \nabla^{l} \nu\right) d \mu,
$$

such that $f$ is smooth and has a polynomial growth, choosing an integer $m$ large enough, the gradient flow of the perturbed functional with $\varepsilon>0$

$$
\mathcal{G}_{m}^{\varepsilon}(\varphi)=\mathcal{G}(\varphi)+\varepsilon \mathcal{F}_{m}(\varphi)
$$

does not develop singularities. This is achieved choosing $m$ so that the rescaling order of $\left|\nabla^{m} \nu\right|^{2}$ is larger than the rescaling order of $f\left(\varphi, g, \mathrm{~A}, \nu, \ldots, \nabla^{s} \mathrm{~A}, \nabla^{l} \nu\right)$, in this way the extra terms coming from $\mathcal{G}$ are well controlled by the leading term in the first variation of $\varepsilon \mathcal{F}_{m}$ and do not affect long time existence.

We say that $\mathcal{F}_{m}$ is a smoothing term for $\mathcal{G}$.

Once we have a sufficiently general family of smoothing terms we can study what happens varying the parameters, in particular when the constant in front of them goes to zero.

This program, suggested by De Giorgi's in [9, 10], Sec. 5, can be stated as follows: given a geometric functional $\mathcal{G}$ defined on submanifolds of the Euclidean space (or a more general ambient space),

- find a functional $\mathcal{F}$ such that the perturbed functionals $\mathcal{G}_{\varepsilon}=\mathcal{G}+\varepsilon \mathcal{F}$ give rise to smooth flows; 
- study what happens when $\varepsilon \rightarrow 0$, in particular, the existence of a limit flow and in this case its relation with the gradient flow of $\mathcal{G}$ (if it exists, smooth or singular).

Our work shows that the functionals $\mathcal{F}_{m}$ satisfy the first point for geometric functionals on hypersurfaces in $\mathbb{R}^{n+1} \mathcal{G}$ with polynomial growth, provided we choose an order $m$ large enough (depending on $\mathcal{G}$ ).

Concerning the second point, a first step would be to consider the possible limits when $\varepsilon \rightarrow 0$ of the flows of $\int_{M} 1+\varepsilon\left|\nabla^{m} \nu\right|^{2} d \mu$ when $m>[n / 2]$ and their relation with the mean curvature flow. Even the simplest case of the convergence of the family of flows of curves associated to the functionals

$$
\mathcal{F}_{1}^{\varepsilon}(\gamma)=\int_{\mathbb{S}^{1}} 1+\varepsilon k^{2} d s
$$

to the mean curvature flow is an open problem.

9.4. De Giorgi's Conjecture. Finally we introduce the original smoothing terms suggested by De Giorgi in [9, 10]. Given a smooth embedded hypersurface $M \subset \mathbb{R}^{n+1}$, we can consider the squared distance function $\eta^{M}(x)=[d(x, M)]^{2}: \mathbb{R}^{n+1} \rightarrow \mathbb{R}$ which turns out to be smooth in a neighborhood of the hypersurface $M$. Then we define the function

$$
A^{M}(x)=\frac{|x|^{2}-\eta^{M}(x)}{2}
$$

and its derivatives

$$
A_{i_{1} \ldots i_{m}}^{M}(x)=\frac{\partial^{m} A^{M}(x)}{\partial x_{i} \ldots \partial x_{m}}
$$

whenever they exist, in particular for every $x \in M$.

The quantities $A_{i_{1} \ldots i_{m}}^{M}(x)$ for $x \in M$ are related to the second fundamental form $\mathrm{A}(x)$ of $M$ and to its derivatives up to the order $m-3$, for instance

$$
\left|A_{i j k}^{M}(x)\right|^{2}=\sum_{1 \leq i, j, k \leq n+1}\left[A_{i j k}^{M}(x)\right]^{2}=3|\mathrm{~A}(x)|^{2} .
$$

In general there is a bijective relation between the quantities $A_{i j k}^{M}(x)$ and the second fundamental form of $M$ at $x$ (see [2]). In the case of immersed manifold, not necessarily embedded, the function $A^{M}(x)$ can be defined using the property that every immersion is locally an embedding.

The relations of the distance function with the second fundamental form make it a valuable tool in the study of the evolution by mean curvature (see [3, 37]) and more in general of geometric functionals and flows (see for instance [2, 11, 12).

De Giorgi suggested that the gradient flow of the functionals

$$
\mathcal{D} \mathcal{G}_{m}(\varphi)=\int_{M} 1+\left|A_{i_{1} \ldots i_{m}}^{M}\right|^{2} d \mu
$$

when $m$ is large enough, does not become singular.

By analogy with our work we expect that when $m>\left[\frac{n}{2}\right]+2$ we obtain regularity.

The first variations of these functionals has been studied by Ambrosio and the author in [2], Sec. 5.3: the leading term of the first variation of $\mathcal{D} \mathcal{G}_{m}$ turns out to be a constant multiple of the leading term of $\mathrm{E}_{m-2}$ (see Theorem 3.7)

$$
2 m(-1)^{m} \overbrace{\Delta \Delta \ldots \Delta}^{m-2 \text { times }} \mathrm{H},
$$

moreover, the functional $\mathcal{D} \mathcal{G}_{m}$ has the same rescaling properties of $\mathcal{F}_{m-2}$.

The difficult step in repeating our proof stays in controlling a priori Sobolev and interpolation constants, or more precisely in obtaining inequalities of kind

$$
\left\|A_{i_{1} \ldots i_{k}}^{M}\right\|_{L^{p}(\mu)} \leq C\left\|A_{i_{1} \ldots i_{k+l}}^{M}\right\|_{L^{q}(\mu)},
$$

since the integrals are done on $M$ but the derivatives are taken along all the directions of the ambient space $\mathbb{R}^{n+1}$.

At this moment the original conjecture of De Giorgi remains open. 
9.5. Asymptotic Behavior. An open problem arising from the discussion of the previous section is the question of the uniqueness of the limit hypersurfaces. It is also unknown to the author if actually it can happen that the hypersurface goes to the infinity when $t \rightarrow+\infty$.

To conclude, we mention the problem of classification of the limit points of these flows, or equivalently of the critical hypersurfaces of $\mathcal{F}_{m}$. In his work [30] Polden completely classifies the limit curves of the flow of the functional (1.1), the analogous $n$-dimensional result seems to be a much more difficult task.

\section{REFERENCES}

1. F. J. Almgren, J. E. Taylor, and L. Wang, Curvature driven flows: a variational approach, SIAM J. Cont. Opt. 31 (1993), 387-438.

2. L. Ambrosio and C. Mantegazza, Curvature and distance function from a manifold, J. Geom. Anal. 8 (1998), no. 5, 719-744.

3. L. Ambrosio and H. M. Soner, Level set approach to mean curvature flow in any codimension, J. Diff. Geom. 43 (1996), 693-737.

4. T. Aubin, Some Nonlinear Problems in Riemannian Geometry, Springer-Verlag, 1998.

5. K. A. Brakke, The Motion of a Surface by its Mean Curvature, Princeton Univ. Press, Princeton, N.J., 1978.

6. M. Cantor, Sobolev inequalities for Riemannian bundles, Bull. Amer. Math. Soc. 80 (1974), 239-243.

7. __ Sobolev inequalities for Riemannian bundles, Differential Geometry, Proc. Sympos. Pure Math., vol. 27, Amer. Math. Soc., 1975, pp. 171-184.

8. P. T. Chruściel, Semi-global existence and convergence of solutions of the Robinson-Trautman (2-dimensional Calabi) equation), Comm. Math. Phys. 137 (1991), no. 2, 289-313.

9. E. De Giorgi, Congetture riguardanti alcuni problemi di evoluzione. A celebration of John F. Nash, Jr., Duke Math. J. 81 (1996), no. 2, 255-268.

10. - Congetture riguardanti alcuni problemi di evoluzione. A celebration of John F. Nash, Jr., CvGmt Preprint Server - Scuola Normale Superiore di Pisa, http://cvgmt.sns.it, 1996.

11. M. Delfour and J.-P. Zolésio, Shape analysis via oriented distance functions, J. Funct. Anal. 123 (1994), 129-201.

12. _ Shape analysis via distance functions: local theory, Boundaries, Interfaces and Transitions (M. Delfour, ed.), CRM Proc. Lect. Notes Ser., AMS, 1998.

13. S. Delladio, On hypersurfaces in $\mathbb{R}^{n+1}$ with integral bounds on curvature, Math. Dept. Univ. Trento, preprint (1998).

14. J. Escher, U. F. Mayer, and G. Simonett, The surface diffusion flow for immersed hypersurfaces, SIAM J. Math. Anal. 29 (1998), no. 6, 1419-1433.

15. L. C. Evans and J. Spruck, Motion of level sets by mean curvature I, J. Diff. Geom. 33 (1991), 635-681.

16. M. Gage and R. S. Hamilton, The heat equation shrinking convex plane curves, J. Diff. Geom. 23 (1986), 69-95.

17. S. Gallot, D. Hulin, and J. Lafontaine, Riemannian Geometry, Springer-Verlag, 1990.

18. Y. Giga and K. Ito, On pinching of curves moved by surface diffusion, Comm. Appl. Anal. 2 (1998), no. 3, 393-405.

19. M. A. Grayson, The heat equation shrinks embedded plane curves to round points, J. Diff. Geom. 26 (1987), 285-314.

20. R. S. Hamilton, Three-manifolds with positive Ricci curvature, J. Diff. Geom. 17 (1982), no. 2, $255-306$.

21. G. Huisken, Flow by mean curvature of convex surfaces into spheres, J. Diff. Geom. 20 (1984), $237-266$.

22. __ Asymptotic behavior for singularities of the mean curvature flow, J. Diff. Geom. 31 (1990), 285-299.

23. _ A distance comparison principle for evolving curves, Asian J. Math. 2 (1998), 127-133.

24. G. Huisken and A. Polden, Geometric evolution equations for hypersurfaces, Calculus of variations and geometric evolution problems (Cetraro, 1996), Springer, Berlin, 1999, pp. 45-84.

25. T. Ilmanen, Elliptic regularization and partial regularity for motion by mean curvature, Mem. Amer. Math. Soc., vol. 108(520), AMS, 1994.

26. E. Kuwert and R. Schätzle, Gradient flow for the Willmore functional, preprint (1999).

27. J. Langer, A compactness theorem for surfaces with $L_{p}$-bounded second fundamental form, Mathematische Annalen 270 (1985), 223-234.

28. J. H. Michael and L. Simon, Sobolev and mean-value inequalities on generalized submanifolds of $\mathbb{R}^{n}$, Comm. Pure Appl. Math. 26 (1973), 361-379.

29. P. Petersen, Riemannian Geometry, Springer, 1998.

30. A. Polden, Closed curves of least total curvature, Arbeitsbereich Analysis Preprint Server - Univ. Tübingen, http://poincare.mathematik.uni-tuebingen.de/mozilla/home.e.html, 1995.

31. _ Curves and Surfaces of Least Total Curvature and Fourth-Order Flows, Ph.D. thesis, Mathematisches Institut, Univ. Tübingen, 1996, Arbeitsbereich Analysis Preprint Server - Univ. Tübingen, http://poincare.mathematik.uni-tuebingen.de/mozilla/home.e.html.

32. _ Compact surfaces of least total curvature, Arbeitsbereich Analysis Preprint Server - Univ. Tübingen, http://poincare.mathematik.uni-tuebingen.de/mozilla/home.e.html, 1997. 
33. L. Simon, Lectures on Geometric Measure Theory, Proc. Center Math. Anal., vol. 3, Australian National University, Canberra, 1983.

34. _ Existence of surfaces minimizing the Willmore functional, Comm. Anal. Geom. 2 (1993), 281-326.

35. G. Simonett, The Willmore flow near spheres, Differential Integral Equations (to appear).

36. J. Simons, Minimal varieties in Riemannian manifolds, Ann. of Math. 88 (1968), 62-105.

37. H. M. Soner, Motion of a set by the curvature of its boundary, J. Diff. Equations 101 (1993), no. 2, 313-372.

38. Y. Wen, Curve straightening flow deforms closed plane curves with nonzero rotation number to circles, J. Diff. Equations. 120 (1995), 89-107.

39. T. J. Willmore, An Introduction to Riemannian Geometry, Clarendon Press, Oxford, 1993.

(Carlo Mantegazza) Scuola Normale Superiore, Pisa, 56126, Italy

E-mail address, C. Mantegazza: mantegaz@sns.it 